\title{
LE BLUET NAIN SAUVAGE
}

Georges-Noël FORTIN

Assistant en horticulture

Normandin, P.Q.

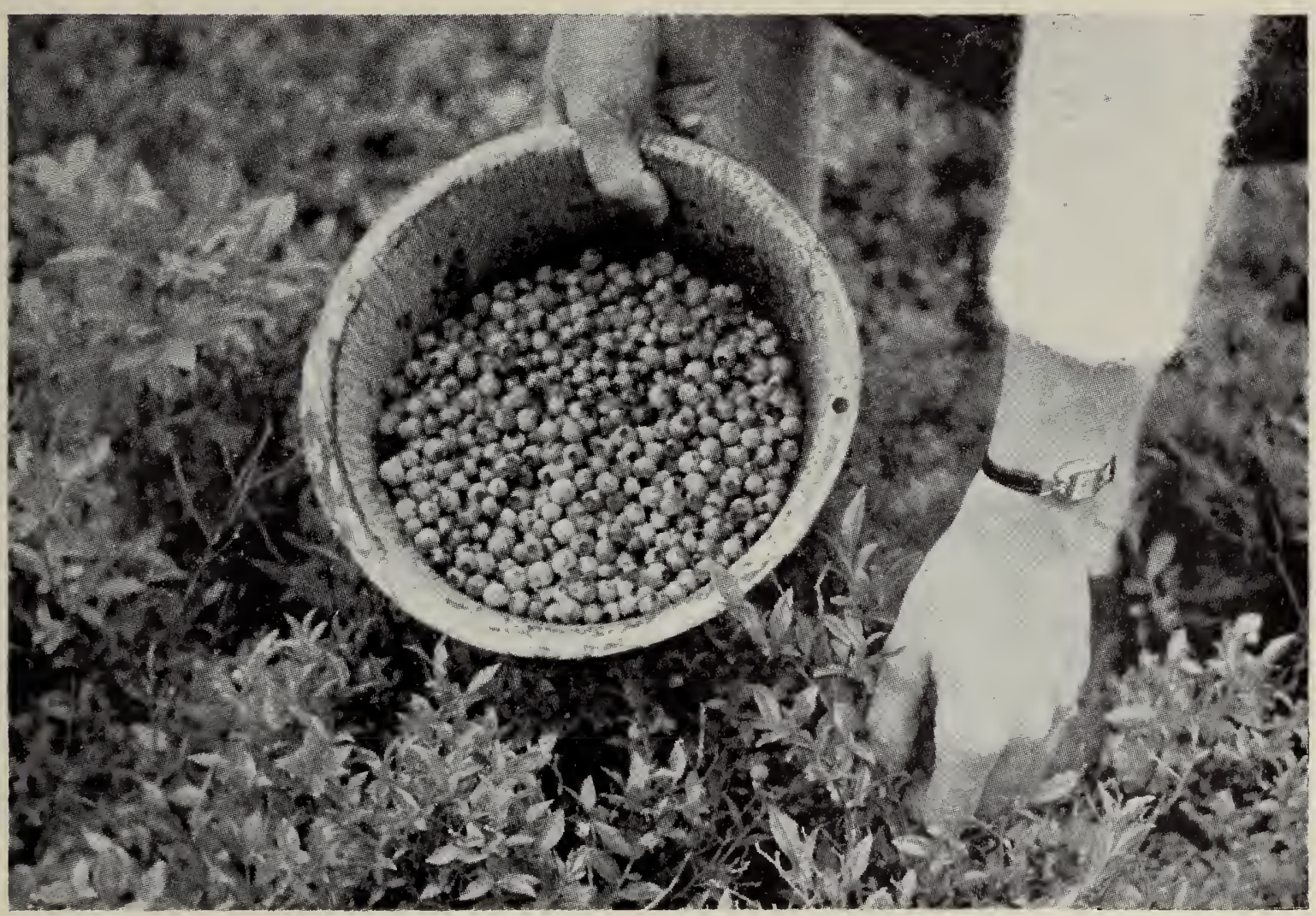

Cueilleur à l'ouvre dans le Québec. L’apparence grise des bluets, sur la photo, correspond à leur couleur naturclle bleu clair.

(Courtoisie du "Bulletin des Agriculleurs")

\section{SERVICE DES FERMES EXPERIMENTALES}

Ministère de l'Agriculture 


\section{REMERCIEMENTS}

L'auteur tient à exprimer sa gratitude envers les ministères de la Colonisation et de l'Agriculture de la Province de Québec sans la collaboration desquels il eut été difficile de procéder à cette étude de la production des bluets. 


\section{SOMMAIRE}

En présentant, sur la production des bluets nains sauvages, des faits et des statistiques peu connus du public, ce bulletin vise avant tout à diffuser les bonnes méthodes de cueillette et de vente de ces petits fruits qui ont la faveur d'un vaste marché en Amérique. Il insiste en particulier sur certaines recommandations faites aux cueilleurs et aux expéditeurs, et qui, si elles étaient mieux observées, pourraient beaucoup pour l'amélioration de cette production.

En effet, il est inutile de songer à la culture des immenses champs de bluets, dans le Québec et ailleurs, si les producteurs ne réalisent pas l'urgente nécessité d'offrir des bluets de première qualité et bien conservés dans des emballages propres et attrayants, et s'ils ne prennent pas les moyens indiqués pour y arriver. La collaboration du cueilleur est la première grande condition: il doit apprendre à manipuler les bluets avec plus de soin et respecter les règlements en vigueur.

Quant aux expéditeurs, il leur faudra moderniser leurs méthodes, populariser des emballages qui plaisent aux clients en même temps qu'ils protègent bien les fruits, et poursuivre certaines expériences entreprises sur le refroidissement et le réemballage des bluets. En tout et partout, ils ont intérêt à coopérer avec les autorités chargées d'appliquer les règlements et d'éduquer le cueilleur. 
Digitized by the Internet Archive in 2012 with funding from

Agriculture and Agri-Food Canada - Agriculture et Agroalimentaire Canada 


\section{TABLE DES MATIÈRES}

Page

INTRODUCTION

PARTIE I-PARTICULARITÉS BOTANIQUES ET

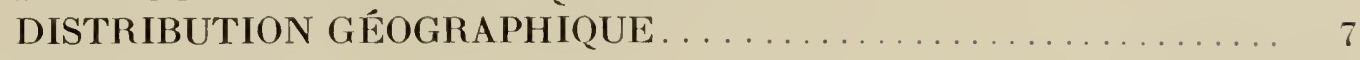

Espèces naines. . . . . . . . . . . . . . . . . . . . . . . . 7

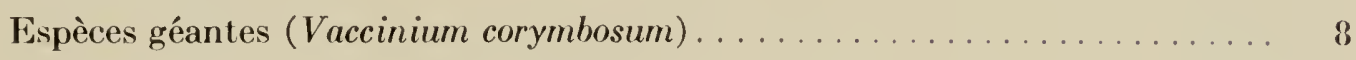

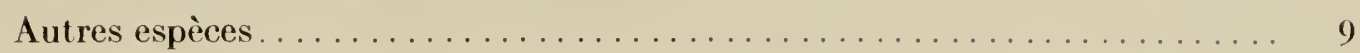

PARTIE II-STATISTIQUES DE LA PRODUCTION DES BLUETS . . . . . . . . 10

La production du Québec......................... 10

PARTIE III-CUEILLETTE, PREPARATION ET VENTE DES BLUETS . . . . . . 16

La cueillette ............................ 16

Saison de cueillette ........................ 16

Usage du peigne .......................... 17

Nettoyage des bluets....................... 17

L'emballage ............................... 18

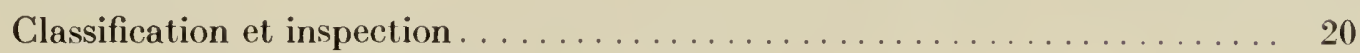

Amélioration de la qualité des bluets. . . . . . . . . . . . . . 21.

Recommandations. . . . . . . . . . . . . . . . . . . . . 23

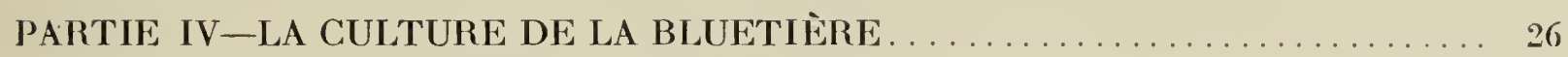

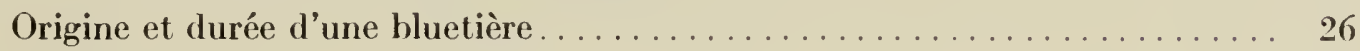

Brûlage. .................................... 28

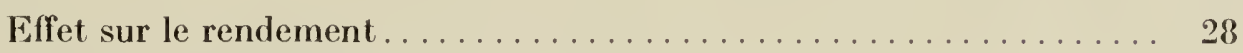

Pratiques populaires. . . . . . . . . . . . . . . . . . . . . . 29

A l'automne ou au printemps. . . . . . . . . . . . . . . . 30

Fréquence du brûlage. . . . . . . . . . . . . . . . . . 30

Recommandations......................... 30

Conclusion. .............................. 31

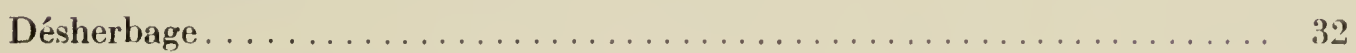

Les mauvaises herbes. . . . . . . . . . . . . . . . . . . . 32

Dommages causés. . . . . . . . . . . . . . . . . . . 32

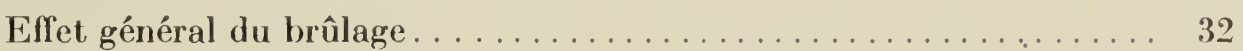

Méthodes de destruction . . . . . . . . . . . . . . . . 33

Maladies et insectes. ........................... 35

Fertilisation . . . . . . . . . . . . . . . .

Le sol à bluet . . . . . . . . . . . . . . . . . . . . . . . 35

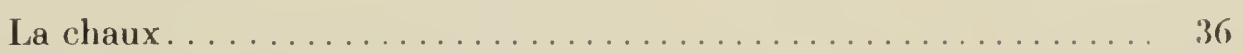

Engrais chimiques........................ 36 


\title{
PERSONNEL TECHNIQUE STATION EXPÉRIMENTALE NORMANDIN, QUÉ.
}

\author{
Adhémar Belzile, B.S.A.
}

Régisseur.

J.-E. Laplante, B.S.A., M.Sc.

Adjoint, céréales et zootechnie.

G.-N. Fortin, B.A., B.S.A.

Adjoint, horticulture.

(a résigné en 1946).

G. Provencher, B.A., B.S.A.

Surveillant, stations

de démonstration.

C.-E. Ouellet, B.A., B.S.A.

Adjoint, horticulture,

apiculture et aviculture.

L.-C. Larose, B.A., B.Sc. (Agr.)

Adjoint, plantes fourragères

et grande culture. 


\section{INTRODUC'TION}

Il est difficile d'ignorer l'importance économique assez grande de la récolte des bluets au cours de la dernière guerre. En raison d'une très forte demande sur le marché des Etats-Unis, la cueillette commerciale des bluets est devenue considérable au Canada, et particulièrement dans la province de Québec.

Pour certaines régions du Québec, ce vaste marché a eu une répercussion très marquée sur leur économie générale. S’il est peu de comtés ruraux où l'on ne découvre pas de bluets poussant à l'état naturel, c'est principalement dans les régions formées par les comtés de Roberval, Lac St-Jean et Chicoutimi, Abitibi el Témiscamingue, et Saguenay, que se trouve la grande production. Les champs de bluets ou "bluetières" (*) y sont immenses, et encore les cueilleurs n'en visitentils qu'une bien petite partie. En fait, il est assuré que le commerce ne manipule chaque année qu'une fraction de la récolte totale possible si l'exploitation des bluetières était poussée d'une façon systématique.

Elles ne sont pas rares, au Lac St-Jean, par exemple, les familles qui ont cueilli 500 boîtes de bluets ( 22 livres par boîte) à chaque saison. Cela leur rapportait, selon les prix courants, de 1,000 à 2,500 dollars. Le bluet devenait ainsi une récolte-argent des plus appréciables pour le cultivateur ou le colon. Le cultivateur pouvait y mettre à profit toute sa main-d'oeuvre familiale et repérer, en l'espace de quelques semaines, un revenu additionnel précieux pour l'amélioration de son exploitation et des conditions de vie sur la ferme. Et en pays de colonisation, plusieurs jeunes paroisses ont pu, grâce à la cueillette des bluets, s'organiser plus rapidement et les colons subsister sur des terres n'offrant que peu de promesses immédiates au point de vue agricole.

Située au coeur de ces immenses bluetières-districts de Dolbeau, Normandin, St-Félicien, Roberval et L'Ascension-, la Station expérimentale fédérale de Normandin, dès sa fondation en 1936, s'est intéressée à la production des bluets et ses possibilités. Elle a collaboré avec les ministères provinciaux de l'Agriculture et de la Colonisation à l'exécution d'un programme d'orientation, de propagande et de recherches: orientation et propagande dans le but de faire l'éducation des cueilleurs et des acheteurs de bluets et les amener à mettre sur le marché un produit de toute première qualité; recherches en vue de l'exploitation systématique ou culture des bluetières comme moyen de régulariser cette source de revenu.

Le présent bulletin contient (a) une brève étude de la distribution géographique des espèces de bluets sur le continent nord-américain et leurs caractéristiques principales; (b) une revue des statistiques de la récolte du bluet nain sauvage (pour le Québec, les chiffres ont été compilés par le Bureau des Statistiques du ministère de I'Industrie et du Commerce, en collaboration avec la Division des Marchés au Service de l'Economie rurale, ministère de l'Agriculture); (c) un chapitre sur la cueillette, la préparation et l'expédition des bluets; (d) enfin, une revue aussi complète que possible de la littérature accumulée à la Station expérimentale de Normandin sur le bluet nain sauvage et la culture de la bluetière. ${ }^{*}$ )

\footnotetext{
(*) Dans le Québec, en langage populaire, on orthograpie “bluet" et "beluet". La forme "bleuet" tend à se répandre dans certains milieux, mais elle ne correspond pas à l'usage des botanistes qui ont exploré la flore québécoise. Bluet, qui semble donc préférable, n'est toutefois pas un canadianisme, contrairement à ce que l'on croît. Mais bluetièr pourrait en être un. Ce terme désigne bien, en tout cas, les savanes, les brôlis, et eul général, tout terrain où le bluet pousse naturellement. L'auteur écrira donc librement "bluet" et "bluetière" au cours de cet ouvrage. (17) (*) Les chiffres donnés entre parenthèses dans le texte de ce bulletin renvoient à la liste des ouvrages consultés paraissant à la firn.
} 


\section{PARTIE I}

\section{PARTICULARITÉS BOTANIQUES ET DISTRIBUTION GEOGRAPIIQUE}

En botanique, le bluet appartient à une grande famille de plantes des climats tempérés et chauds, les Ericacées, famille qui comprend l'airelle, la pyrole, le lédon, la Gaylussacia, le petit thé, la rose-marie, la fleur-de-mai, le thé des bois, le raisin d'ours, le crevard de moutons (bois chaud ou Kalmia), et autres. Les airelles constituent le genre Vaccinium, et les plus connues d'entre elles sont l'airelle-atocas ou atocas (pomme de terre) et l'airelle-bluet ou bluet tout court.

La distribution géographique du bluet et de ses nombreuses espèces sur le continent nord-américain illustre à merveille la façon dont les plantes, en général, se différencient en s'adaptant à diverses conditions de climat et de sol. L'on s'en tiendra ici à une brève description de sept espèces principales qui ont une valeur économique propre. (7). On les divise entre elles, selon leur taille, en bluets nains (lowbush blueberry), qui mesurent de 6 à 18 pouces de hauteur, et en bluets géants (highbush blueberry).

\section{Espèces naines}

La quasi-totalité des bluets (fruits) vendus sur le marché provient de deux espèces naines. La première, et la plus répandue selon le botaniste Gray, est le Vaccinium angustifolium (Ait.) ou $V$. pennsylvanicum Lam., bluet de Pennsylvanie. On la reconnaît à son fruit sphérique, bleu clair, très sucré, avec ou sans pruine (*), à ses feuilles lisses et luisantes aussi bien à l'endroit qu'à l'envers. Une variété de cette espèce porte des fruits noirs et généralement sans pruine qu'on rencontre souvent parmi les types bleus. ( $V$. p. nigrum (Wood).

La deuxième espèce, $V$. canadense Kalm, est plus tardive que la précédente, d'après Gray encore. Marie-Victorin (13) précise qu'elle fleurit un peu après le $V$. pennsylvanicum dans le sud du Québec, mais qu'au Lac St-Jean les deux fleurissent ensemble. Son fruit est plutôt petit, bleu clair avec pruine et ses feuilles et sa tige sont densément duveteuses.

Certains rapportent avoir vu souvent des bluets blancs. Il en existe, mais ce ne sont pas des espèces distinctes. Il s'agit de variétés albinos des expèces ordinaires. (*) Un auteur assez ancien attribuait ce phénomène parfois à la présence de champignons (fungi). (16).

L'aire géographique où les bluets de Pennsylvanie et du Canada croissent en commun est très vaste. Elle comprenid l'Ile de Terre-Neuve, le Labrador, le Québec entier, le nord de l'Ontario, les Provinces Maritimes, et aux Etats-Unis, les Etats de Maine, New-York, Michigan et Vermont. Marie-Victorin (13) soutient, "d'après des expériences précises", que ces espèces "bien qu'adaptées au climat du nord ne sont pas vraiment résistantes à la gelée". Si elles ne succombent pas d'ordinaire aux durs hivers de l'Abitibi et du Lac St-Jean, par exemple, c'est grâce à leur petite taille qui permet à la couche de neige, habituellement épaisse, de les recouvrir entièrement et de les protéger contre le grand froid. On a pu constater qu'en 1944, après un hiver remarquablement dénué de neige, la récolte fut à peu près nulle en Abitibi et très inférieure à la normale au Lac St-Jean. La cueillette ne valait la peine que dans les endroits moins exposés aux grands vents, là où un peu de neige avait pu s'accumuler.

(*) On appelle pruine ou pruinosilé cette matiré grenue, cireuse, qui recouvre un grand nombre de fruits et légumes, prunes, bluets, choux, etc., et aussi certains champignons. Elle leur donne une apparance grisâtre.

(*) L'albinisme est un phénomène génétique qui se manifeste par l'effacement des pigments naturels (couleur) et qui se rencontre aussi bien dans le règne végétal que dans le règne animal. 


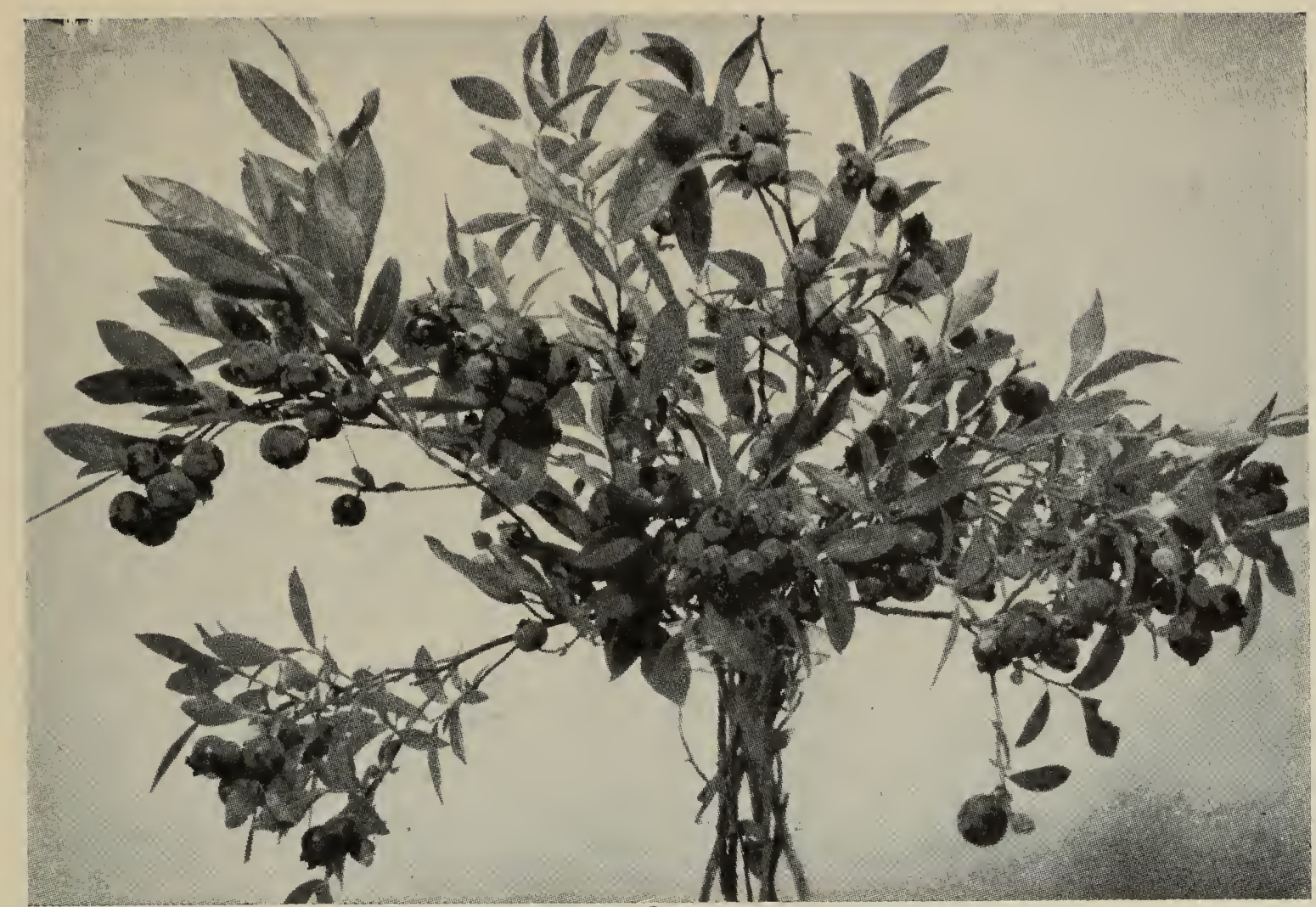

Fig. 1.-Bouquet de bluets nains du Lac St-Jean, province de Québec.

(Courtoisie du "Bulletin des Agriculteurs")

\section{Espèces géantes}

Le bluet en corymbe. La troisième espèce, en importance, est le bluet géant ou airelle en corymbe (highbush blueberry), Vaccinium corymbosum L. (V. australe), qui peut croître jusqu'à 10 ou 15 pieds de hauteur. Son fruit ressemble beaucoup à celui du bluet nain.

Ce bluet géant résiste moins à la sécheresse que le bluet nain. C'est pourquoi il préfère la côte de l'Atlantique, le nor'd de la Floride jusqu'au sud du Maine, et les bords des Grands-Lacs. Il s'en trouve quelques colonies dans la région de Montréal. En Nouvelle-Ecosse, les comtés de Digby, Yarmouth et Shelburne sont les seuls où cette espèce soit répandue. La haute taille du bluet en corymbe l'exclut définitivement des régions nordiques où, à défaut d'autre protection naturelle, la couche de neige ne suffit pas à le défendre contre le froid rigoureux. On a constaté qu'il succombe à une température de 20 degrés F. sous zéro. (7). Des plants de bluets géants recueillis dans les boisés de Drummondville, St-Majorique et St-GérardMajella, dans le Québec, et transplantés à Normandin, Lac St-Jean, n’ont pas survécu à l'hiver dans leur's parties non protégées par la neige, pas plus d'ailleurs que certaines variétés importées de bluet géant amélioré.

Plusieurs sélections du bluet géant sauvage ont été faites aux Etats-Unis, depuis 1906. Le travail d'amélioration a tellement bien été poussé depuis qu'on peut compter plus de 25 variétés cultivées de bluets géants, en grande partie or'iginaires de l'Etat de New-Jersey. La Colombie-Britannique et la NouvelleEcosse en ont créé plusieurs nouvelles variétés. A la Station expérimentale fédérale de Kentville, Nouvelle-Ecosse, "à mesure que progresse le travail de croisement et de sélection, il se développera des variétés encore plus résistantes que celles déjà connues" (8). Quelques-unes ont déjà été nommées qui sont issues de croisements entre le bluet nain et le géant. Ces variétés plus résistantes pourraient un jour' s'adapter à certaines régions de la province de Québec et de l'Ontario où la culture du bluet amélioré est à peu près inexistante, si ce n'est à l'état expérimental. 
T'outes ces variétés domment de meilleurs rendements que les plantes sauvages, des fruits plus gros et plus uniformes. II s'en cultive environ 2,500 acres aux Etats-Unis où la production annuelle varie de 3 à 5 millions de chopines.

Le bluet géant amélioré soulève un intérêt considérable parmi les chercheurs c. les horticulteurs. L'étude des exigences au point de vue du sol et du climat a conduit deux auteurs à conclure dans les termes suivants une communication sur l'acidité de la sève du bluet: "Au point de vue physiologique, le bluet (et peutêtre les plantes semblables, l'atocas, par exemple) ne diffère-t-il pas de la majorité des plantes cultivées, et si tel est le cas, est-ce qu'il ne faudrait pas entreprendre d'en étudier profondément la physiologie afin d'élargir notre connaissance du métabolisme végétal?" (11). Aussi la culture de cette plante est-elle considérée comme une haute spécialité. Comme les plants de Vaccinium corymbosum naissent généralement d'une seule couronne, grosse, ligneuse au lieu de se propager par tiges souterraines comme le bluet nain, on doit les inultiplier par boutures, procédé difficile pour lequel on a dû développer une technique spéciale. $\left.{ }^{*}\right)$.

\section{Autres espèces géantes}

Une quatrième espèce de bluct qui ressemble au $V$. corymbosum, sauf pour quelques légères différences, c'est le $V$. pallidum (dryland blueberry), bluet des landes. Cette plante n'existe pas au Canada. Elle croît dans les Etats d'Alabama et de Georgie, et vers le nord jusqu'au Maryland et la Virginie de l'Ouest. Elle est de taille moyenne, souvent appelée "low blueberry" parce qu'elle ne mesure que de 1 à 3 pieds de hauteur. Le fruit est bleu clair et mûrit plus tard que celui des espèces énumérées plus haut. On a aussi travaillé à son amélioration en vue de la culture commerciale.

Le $V$. ovatum, bluet toujours vert (evergreen blueberry), qui s'étend du centre de la Californie à la Colombie britannique, le long de la côte du Pacifique, doit son importance commerciale à son feuillage plutôt qu'à son fruit. Ses branches sont attrayantes et se vendent pour fins de décoration. Quant au fruit, il a une saveur spéciale qui le rend inférieur aux autres types mis sur le marché.

Les monts Cascades, dans l'Orégon, et les montagnes Rocheuses hébergent le bluet de montagne (broadleaf blueberry), $V$. membranaceum. Le plant, très résislant à la sécheresse, mesure de 3 à 5 pieds de hauteur. Le fruit est porté par paires ou même par unité sur le pédoncule, et non pas en grappes comme c'est le cas des autres espèces.

Enfin, on trouve dans la Georgie du sud et dans le nord de la Floride le V. ashei (rabbiteye blueberry) qui passe pour le bluet le plus résistant à la chaleur et à la sécheresse. L'arbuste est très grand. Il peut mesurer de 10 à 15 pieds. Il porte des fruits noirs de qualité médiocre, peu recherchés sur le marché à cause de leur couleur. On en a cependant développé plusieurs variétés de meilleure qualité. Actuellement, la culture se résume à transplanter en rangs dans des endroits favorables des plants choisis dans leur habitat naturel. La superficie ainsi cultivée est d'environ 2,500 acres.

(*) Voir "Le bluet" par E.-L. Eaton el al (Ottawa, ministère de l'Agriculture. Publication no 754, 1944). 


\section{PARTIE II}

\section{S'TA'TISTIQUES DE LA PRODUCTION DES BLUETS}

La production canadienne de bluets nains sauvages est considérable et alimente, avec celle de l'Ile de Terre-Neuve, le vaste marché américain. En 1943, par exemple, on a estimé que les bluets canadiens exportés se chiffraient à $16,000,000$ de livres, dont 2,210,000 livres à l'état congelé. En 1945, les exportations atteignaient les chiffres records de 22,806,000 livres de bluets frais et 2,608,000 livres de bluets congelés; presque toute la production du Québec (qui était, cette année-là, de 735 chars, ou environ 21,000,000 livres) fut expédiée aux Etats-Unis. Toutes les expéditions canadiennes se composent uniquement des fruits du bluet nain sauvage.

Il faut savoir, cependant, quelle est la production des bluets aux Etats-Unis. En 1944, Darrow et autres $(2,7)$ estimaient la production annuelle comme suit:

Maine: $1,000,000$ pintes ("quarts") et plus;

Michigan, New-Jersey: 500,000 et plus, chacun;

Floride, Caroline du nord, New-York, Alabama et New-Hampshire: 100,000 et plus; Pennsylvanie, Ohio, Indiana, Wisconsin, Missesota, Iowa, Louisiane, Washington, Virginie de l'Ouest, Vermont et Mississipi: 1,000 et plus.

L'Ile de Terre-Neuve exporte une grande quantité de bluets congelés. Le tableau I permet de comparer l'importance relative de Terre-Neuve et du Canada sur le marché des bluets frais et des bluets congelés. Ces chiffres ont été compilés par les compagnies de chemin de fer.

Pour les provinces canadiennes autres que Québec, des relevés statistiques ammuels ne sont pas disponibles. Eaton (9) admet que l'industrie des bluets n'est pas très développée dans les Provinces Maritimes. En Nouvelle-Ecosse, le comté de Cumberland est le plus gros expéditeur. La superficie des bluetières de cette province était estimée à 43,000 acres environ, en 1931. Cette province a expédié 46,000 cageots de bluets frais, en 1930, principalement vers Boston et New-York. Cette quantité diminua au cours des années suivantes, alors que furent interdites les expéditions de fruits infestés par les vers. L'industrie des conserves y absorbe aujourd'hui une quantité croissante de fruits.

Au Nouveau-Brunswick, la production est plus considérable. Le seul comté de Gloucester en a vendu 2,500,000 livres en 1939, sur une production totale de $4,000,000$ livres pour toute la province. Une certaine quantité de bluets est congelée avant l'expédition. On en expédie beaucoup également à l'état frais vers les conserveries du Maine (9).

Le commerce et l'industrie des bluets se développent lentement sur l'Ile-duPrince-Edouard. En 1930, les expéditions furent de 200,000 livres seulement. Dix ans plus tard, en 1940, 600,000 livres de bluets congelés étaient expédiées de l'Ile vers le centre du Canada et aux Etats-Unis.

Le nord de la province d'Ontario comprend de vastes bluetières où s'alimente en partie le marché de Toronto.

La cueillette des bluets n'a pas de grande importance commerciale dans les provinces de l'Ouest.

\section{La production de Québec}

Le province de Québec est, sans contredit, celle d'où vient la plus grande partie de la production canadienne de bluets. On y trouve plusieurs conserveries et congélateurs à blucts. Montréal est le grand centre d'expédition de bluets frais et congelés venant de toutes les provinces, particulièrement de la province de Québec. 
Pour la produclion des bluels, on divise la provinee de Québec en qualre régions qui sonl, par ordre diumporlance: (a) Lac St-Jean-Chicoutimi, (b) Abitibi-Témis-

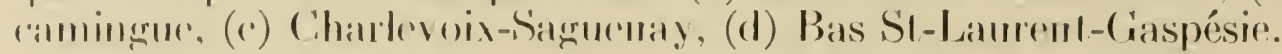

Le région du Iar Si-Jean-Chicoulimi a loujours élé la plus grande productrié de bluets. Les principaus rentres d'expédilion soml Dolbeau, Normandin, StFélicien. Roberval. L'Aseension ol Chicontimi. La eongélation, la déshydratation, lo prérefroidissement of le lransport par avion y ont été l'objet de quelques expériences, mais loule la prednction commerciale y est encore expédiée à l'état frais en wagons réfrigérés. Une partie des expéditions de Charlevoix-Saguenay est ronfiéc aux bateaux.

L'Abilibi-Témiscamingue n'a fail sa première apparition importante dans ce domainc qu'en 1943, après de grands feux de forêt. Presque toute la récolte de rette région est dirigée vers Toronto, alors que celle du reste de la province passe par Montréal. Le Bas St-Laurent et la Gaspésie sont les seuls endroits encore à faire la congélation des blucts avant expédition. Aujourd'hui, 95 pour cent environ des blucts de cette région sont expédiés à l'état congelé.

II n'y a de statistiques provinciales officielies que pour les récoltes de 1942 et suivantes. Pour les années précédentes, des rapports compilés par les Chemins de fer Nationaux donnent une idée du volume ordinaire des expéditions pour la province, soit environ 3.000,000 livres. La récolte de 1935 fut de 5,000,000 livres. Celle de 1941 fut de 6,286,445.

Les statistiques officielles des récoltes de 1942 et suivantes sont rapportées aux tableaux II et III. Elles sont basées sur les expéditions commerciales seulement, mais elles représentent presque toute la récolte dont une fraction minime seulement est destinée au marché domestique. Par ces tableaux, on voit que la récolte totale de bluets passe de $6,286,445$ livres $(\$ 575,962)$ en 1942 , à 20,120,827 livres $(\$ 2,573,476)$ en 1943 . Cet accroissement peut être rattaché à trois causes au moins: (a) incidence, en 1941, de désastreux feux de forêt au Lac St-Jean et en Abitibi; (b) récoltes à peu près nulles au Maine et dans les Provinces Maritimes; (c) conséquemment, demande considérablement accrue pour les bluets sur le marché américain el la hausse conséquente des prix. On remarque, pendant la même période, que le prix moyen perȩu par le eueilleur passe de 9 à 13 rents la livre (Tableau IV). En 1914, les gelées printanières et la sécheresse de l'été n'onl permis de récolter que 6,665,612 livres de bluets, mais les expéditions valaient quand même $\$ 1,296.821$, ear le prix moyen a été de 19.5 cents la livre. Lannée suivante donna une réeolte record: $21,044.875$ livres évaluées à $\$ 4,373,042$, all prix moyen de 20.8 cents la livre. Pnis, on observe une baisse générale du marché, expéditions el valeurs, avee le retour de bonnes récoltes dans l'Etat du Maine ot à Terre-Neuve. De plus, les routes commerciales libérées par la cessation des hostilités permettent aux Etats-Unis de diversifier leurs importations de fruits. La récolte québécoise est tombée graduellement à 14,610,480 livres en 1946, et 11,437,485 livres en 1947, avec chute correspondante des prix moyens à 11.8 el 11.1 cents la livre. On remarque encore que c'est au Lac St-Jean-où, pourtant, les bluets sont les plus abondants-que les prix sont les plus élevés. Cela semble dû à l'activité commerciale et à la concurrence des acheteurs locaux ayant à satisfaire une très forte demande, et non pas exclusivement aux qualités naturelles du fruit, d`ailleur's inégalées. Au dire du Service provincial d'Inspection, les bluets de l'Abitibi, quoique plus petits et payés moins cher, "classaient" beaucoup mieux que ceux du Lac St-Jean au cours des saisons 1943 et 1914. A noter, enfin, que la majeure partie de la récolte commerciale est faite sur les immenses terres de la Couronne dans le Nord du Québec, ou dans les limites concédées aux compagnies forestières. Un faible volume cueilli sur les terrains privés va en partie à la consommation locale. 


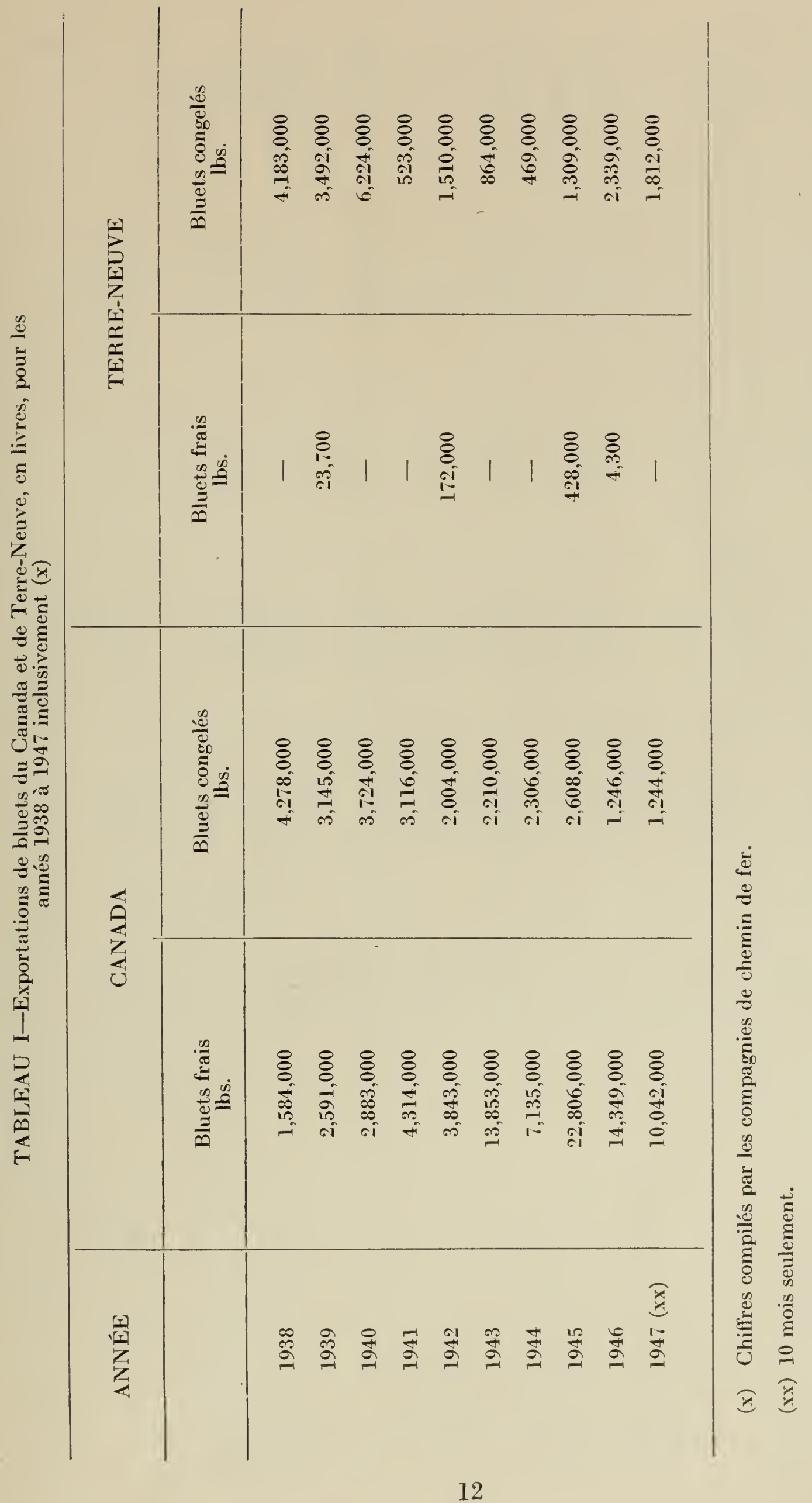




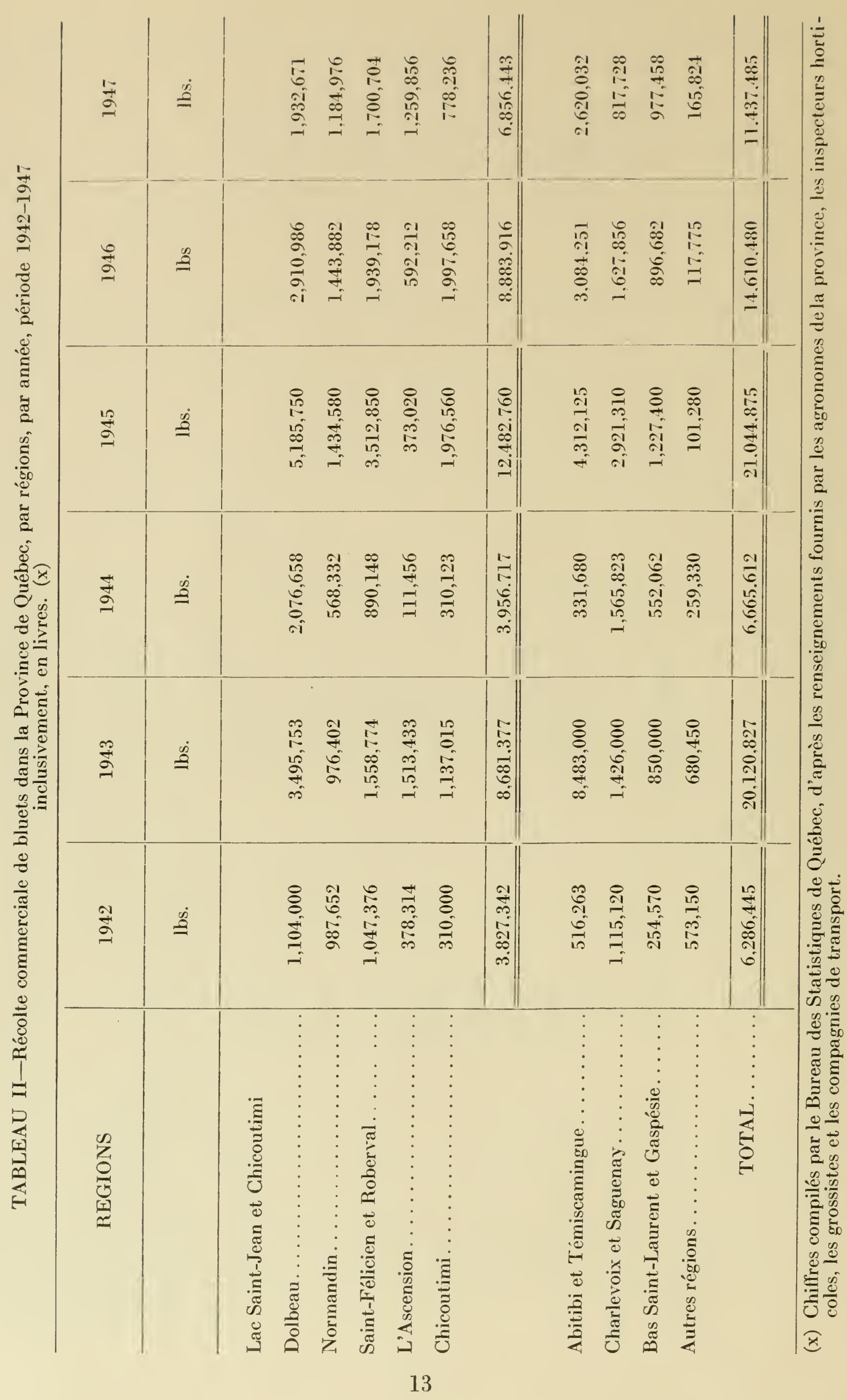




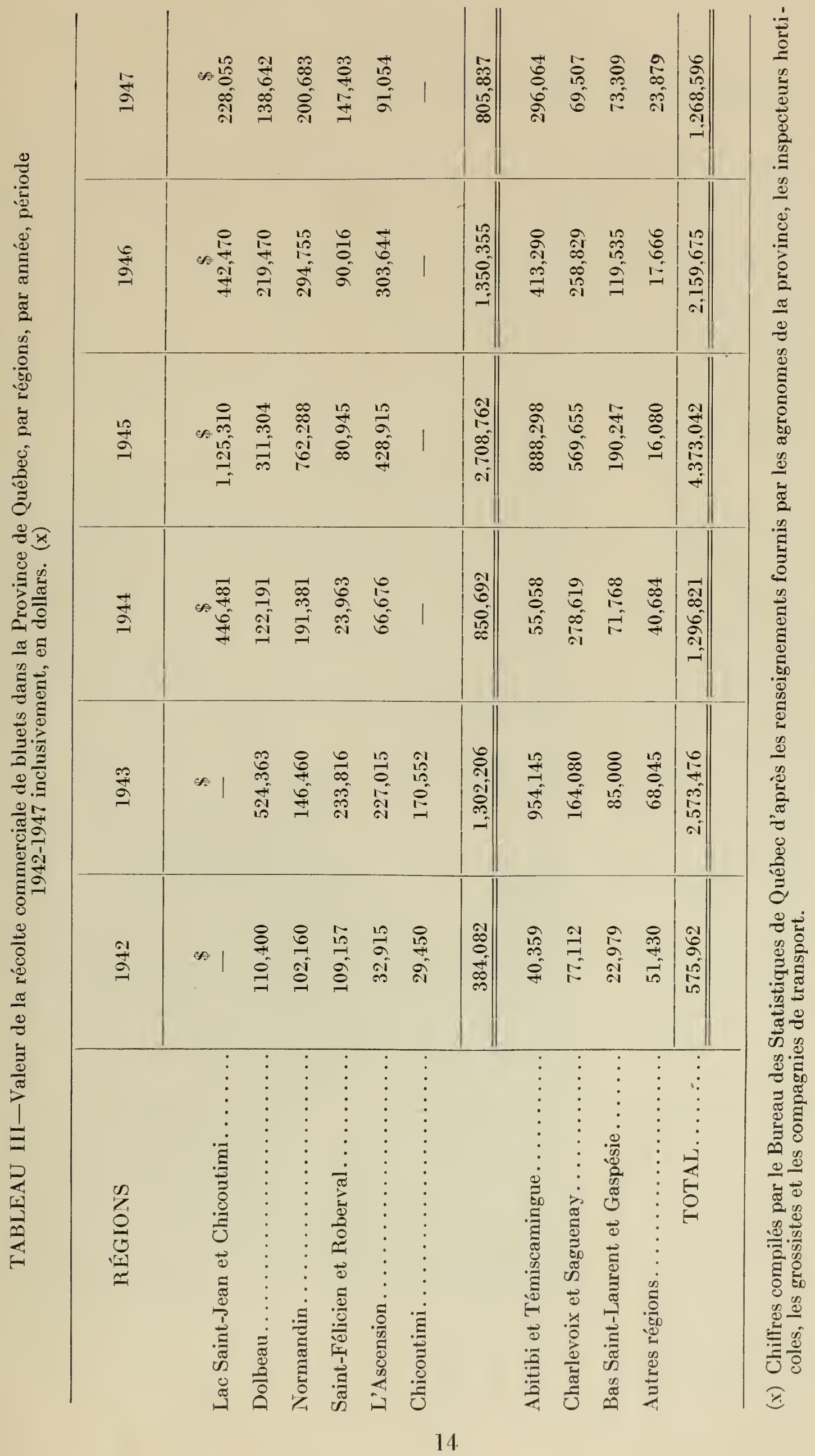




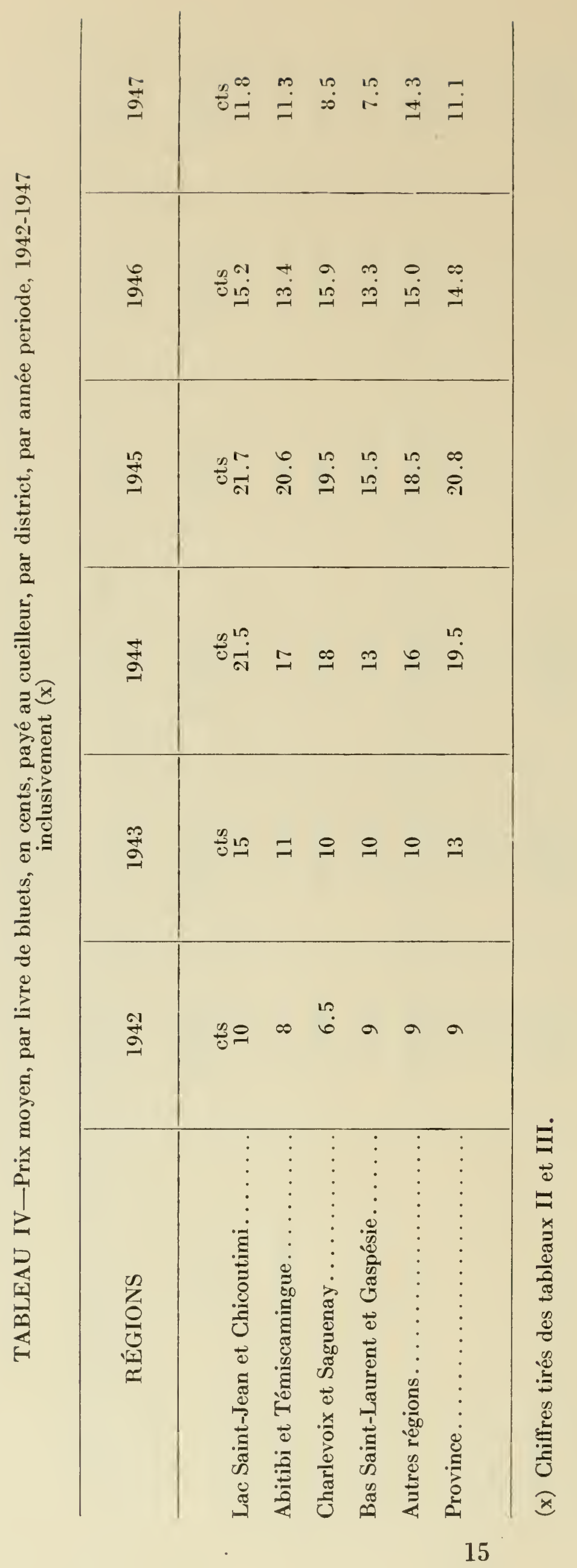




\section{PARTIE III}

\section{CUEILLETTE, PRÉPARATION ET VENTE DES BLUETS}

\section{La cueillette}

Le Vaccinium pennsylvanicum et la $V$. canadense ne se distinguent pas dans les champs, en pratique. Les deux espèces croissent ensemble, bien que la première préfère les terrains acides, tourbières, gneiss laurentien, et la seconde les lieux humides et les bois rocheux. Eaton (9) explique que "ces différents types de bluets se trouvent souvent par groupes, comme si chacun d'eux avait pris naissance dans un semis de hasard et qu'il se serait plus tard propagé par les racines. Cette tendance est surtout apparente lorsque les bluets se propagent dans un champ qui est depuis longtemps défriché. La précocité, le goût, la grosseur, la couleur, la fermeté et la forme du fruit; la densité de la couleur, la forme, la résistance à la maladie de la part du feuillage, et beaucoup d'autres différences s'aperçoivent aisément. Malgré ce mélange de types, de variétés, le bluet supporte l'expédition mieux que la

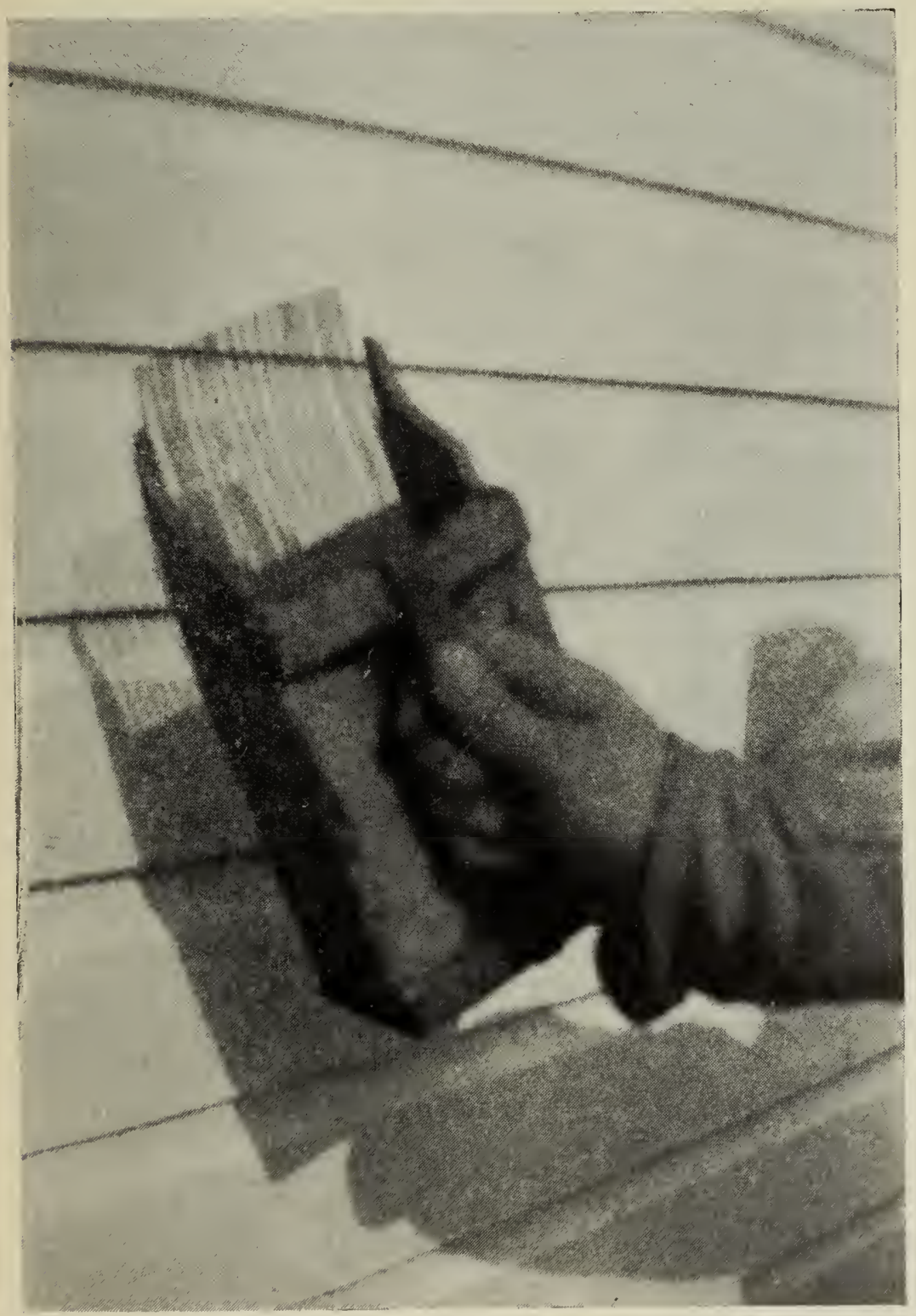

Fig. 2.- "Peigne" de fabrication domestique en usage dans les bluetières du I ac St-Jean. Cet instrument endommage et les fruits et les plants de bluets. p!upart des autres petits fruits".

Saison de cueillette Au Lac St-Jean, et en général dans le Québec, les bluets commencent à mûrir dans les derniers jours de juillet ou durant la première semaine d'août selon les années. On pourra les cueillir jusqu'en septembre, car, à moins d'accidents atmosphériques comme une sécheresse prolongée ou des orages violents, les fruits restent attachés à l'arbuste jusqu'à un état de maturité assez avancée. Entre temps, ils tirent profit des pluies et de l'humidité du sol pour se maintenir à leur pleine grosseur.

Les habitués de la cueillette commencent à affluer dans les bluetières durant la première quinzaine d'août et y demeurent pendant cinq ou six semaines, apportant avec eux provisions de bouche, batterie de cuisine, tentes, etc. Plusieurs s'enfoncent aussi loin qu'à 50 , 75 ou 100 milles de leur foyer. Les premières gelées 
d'automne, vers la mi-septembre, donneront le signal du retour. Chaque soir, les cueilleurs procèdent vers leur campement avec le produit de la cueillette qu'ils nettoient sommairement et mettent en boîtes. Un camionneur vient chaque jour du centre d'expédition le plus rapproché acheter ces bluets et les paie sur-le-champ au nom de l'acheteur qui l'a commissionné.

L'usage du peigne. Dans le Québec, jusqu'en ces dernières années, la cueillette était faite à la main. De plus en plus, maintenant, on se sert du "peigne", instrument très répandu dans les Provinces Maritimes et au Maine dont la récolte est, en majeure partie, destinée aux usines de mise en conserve. C'est une boîte de forme et de dimensions assez variables, ouverte à une extrémité et armée de dents de broche rapprochées d'un huitième de pouce environ, à la manière des dents d'un peigne, d'où le nom. C'est avec cela qu'on "peigne" les grappes de bluets. (Fig. 2)

Ce mode de cueillette est beaucoup plus expéditif que la cueillette à la main. Mais il suscite de nombreux inconvénients. Au Maine, selon Munson (15), "les premiers fruits mûrs sont cueillis à la main, et seulement les fruits mûrs. Plus tard dans la saison, en particulier sur les 'vieux brûlés', c'est-à-dire sur les superficies qui doivent être brûlées l'année suivante, le fruit est ramassé avec le peigne". L'usage du peigne se trouve donc déjà considérablement restreint, et pour plusieurs raisons.

D'abord, la qualité du bluet "peigné" est toujours inférieure à celle du bluet cueilli à la main, même au plus fort de la saison. D'après Chandler (2), "quelque soit le genre de peigne utilisé, on doit toujours prendre soin de ne pas briser ni écraser les bluets. Si seulement 40 pour cent de la "poussière bleue" (pruine) sont enlevés, tout le contenu d'une boîte sera très mouillé à son arrivée sur le marché, Il peut n'en rien paraître le jour même de la cueillette, mais, le lendemain, la chair devient rouge et les bluets perdent du poids et s'affaissent plus rapidement que des bluets sains et fermes".

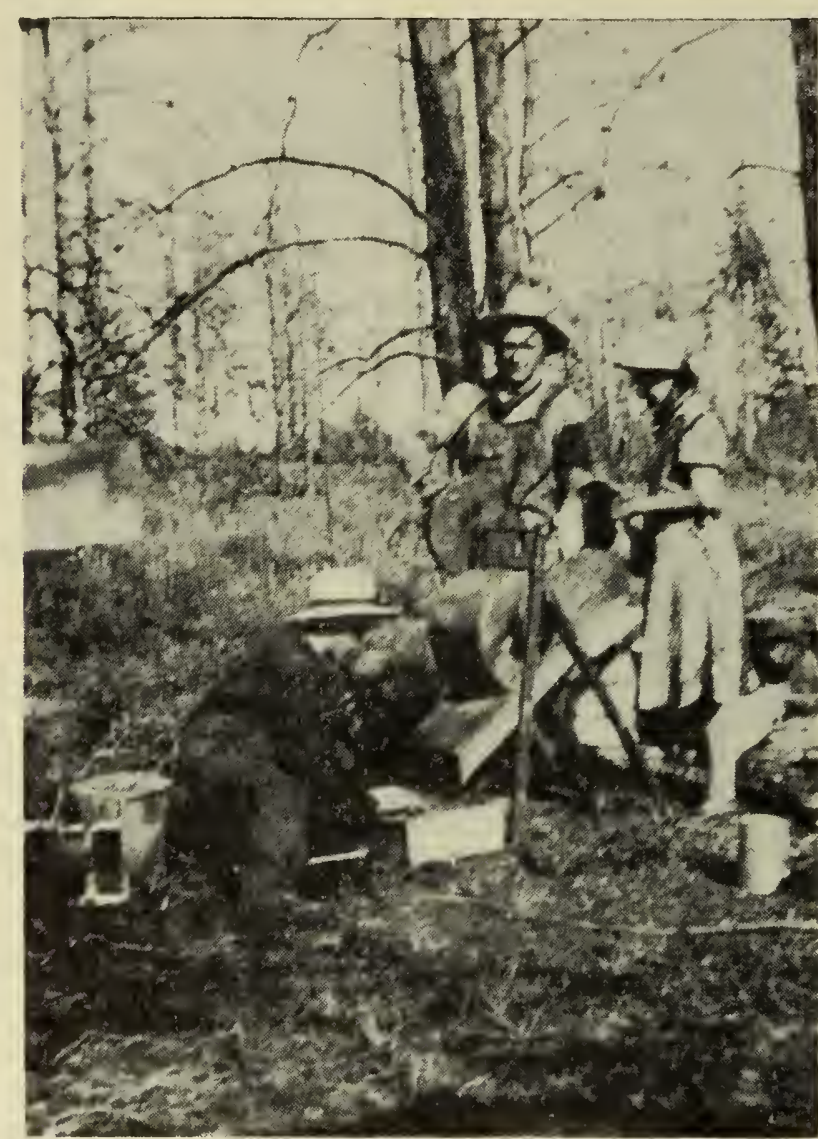

Fig. 3.-Petit crible dont on se sert pour le netloyage des bluets dans le champ. (Lac St-Jean).
On fait aussi remarquer (15) que le peigne endommage le plant dont il brise ou arrache les bourgeons à fruits pour l'année suivante. Les cueilleurs exercés savent reconnaître, à leur faible rendement, les superficies endommagées par le peigne et les négliger pour des bluetières vierges ou déjà cueillies à la main seulement.

Le nettoyage des bluets. A moins qu'on ne cueille les bluets à la main et avec un soin particulier-ce qui se pratique de moins en moins - un vigoureux nettoyage s'impose avant l'emballage. On utilise la ventilation naturelle ou forcée. Dans le premier cas, le cueilleur élève les bluets à hauteur de bras et les laisse tomber lentement dans un récipient placé à ses pieds. Le vent fait dévier feuilles et brindilles vers le sol. Dans le second cas, on se sert d'un crible rudimentaire. On soumet les bluets au courant d'air autant de fois que nécessaire. (Fig. 3 et 4 ) 


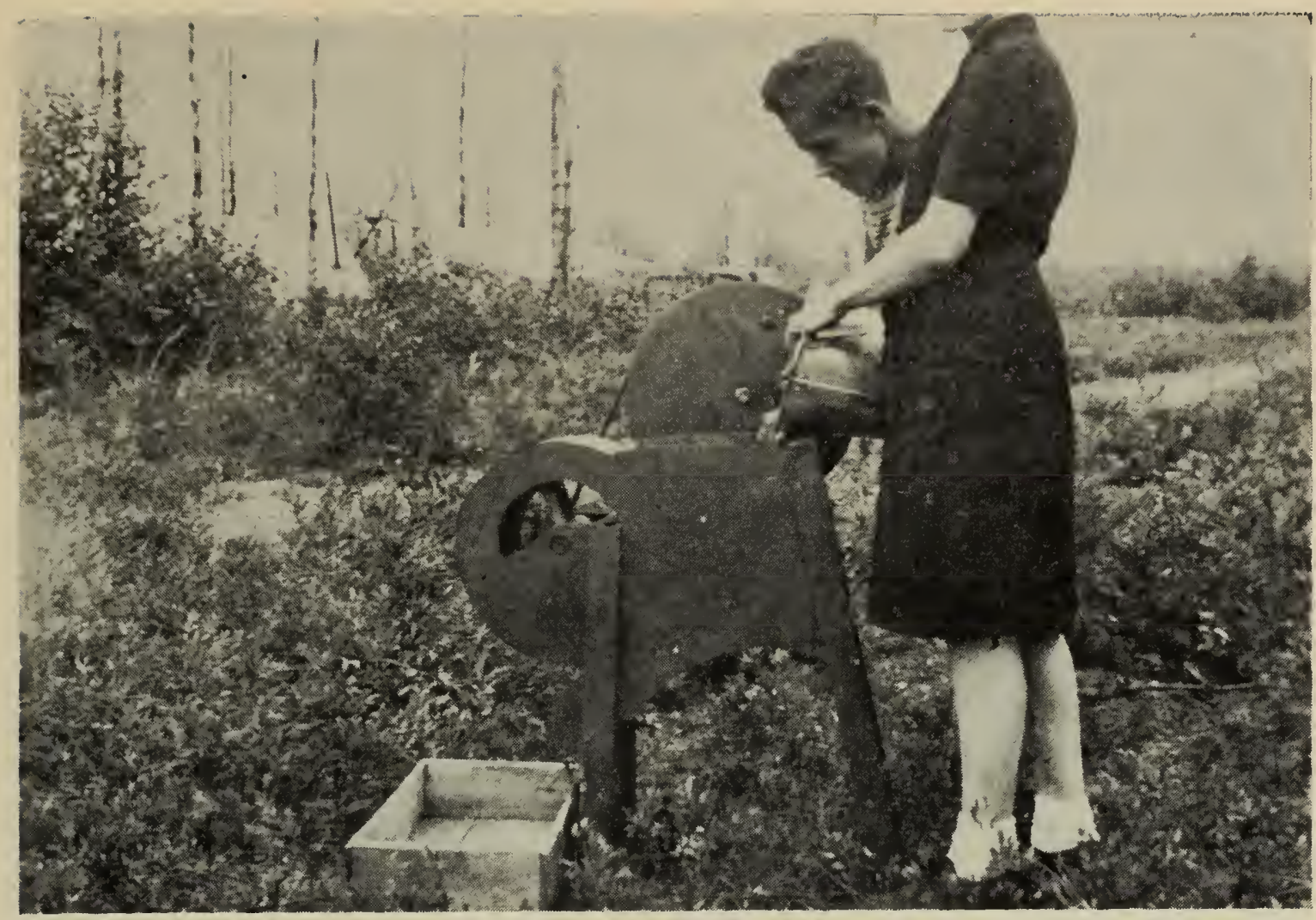

Fig. 4.-Autre genre de crible en usage. (Lac St-Jean).

Le nettoyage est une manipulation que les bluets ne subissent jamais sans y perdre de leur qualité, surtout s'il est fait dans le champ même. Chandler encore (2) affirme que le peigne et le crible, à la façon dont on les utilise au Maine, brisent les fruits dans une proportion de 5 à 20 pour cent.

\section{L'emballage.}

Dans la province de Québec, les bluets sont emballés en casseaux, en paniers et en boîtes. La "Loi des Produits Agricoles et Règlement sur la Vente des Fruits et Légumes" (Québec, Ministère de l'Agriculture, 1937) dit, à ce sujet:

"Seuls sont admis pour les bluets les casseaux de 1 pinte. Les casseaux seront remplis à pleine capacité et les produits seront tassés au moyen de secousses légères et répétées. Les casseaux peuvent être emballés dans des cageots contenant soit 24 ou 32 ou 36 casseaux de même capacité. On peut aussi utiliser un cageot à un seul lit contenant 8 casseaux.

"Il est perınis d'emballer les bluets dans des paniers de 11 pintes ou dans des boîtes de dimensions suivantes:

(a) les boîtes contenant 22 livres de bluets, poids net, devront mesurer à l'extérieur $5 \times 12 \times 24$ pouces, et, à l'intérieur, $4 \times 11 \times 22$ pouces,

(b) celles contenant au moins 30 livres de bluets, poids net, devront mesurer à l'intérieur $5 \frac{1}{2} \times 12 \frac{1}{2} \times 21$ pouces, l'épaisseur des planches des bouts n'excédant pas 1 pouce et les autres planches formant le fond, le couvercle et les autres côtés devant avoir une épaisseur maximum d'un demi-pouce."

Les expéditions de bluets en casseaux sont les moins importantes, et elles se font par train-express. Ces bluets sont destinés à être consommés dès leur apparition sur le marché. Deux variétés de casseaux sont d'usage courant: (a) le casseau de bois, expédié en cageots de 24, 32 ou 36 casseaux, (b) et le casseau de carton ciré, avec couvercle à fenêtres de cellophane, expédiés dans des boîtes de carton fort de 8 ou 16 casseaux. Ces derniers emballages sont de beaucoup les plus appréciés à cause de leur apparence. Leur usage s'est répandu depuis quelques années et devrait être encouragé. (Fig. 5). 


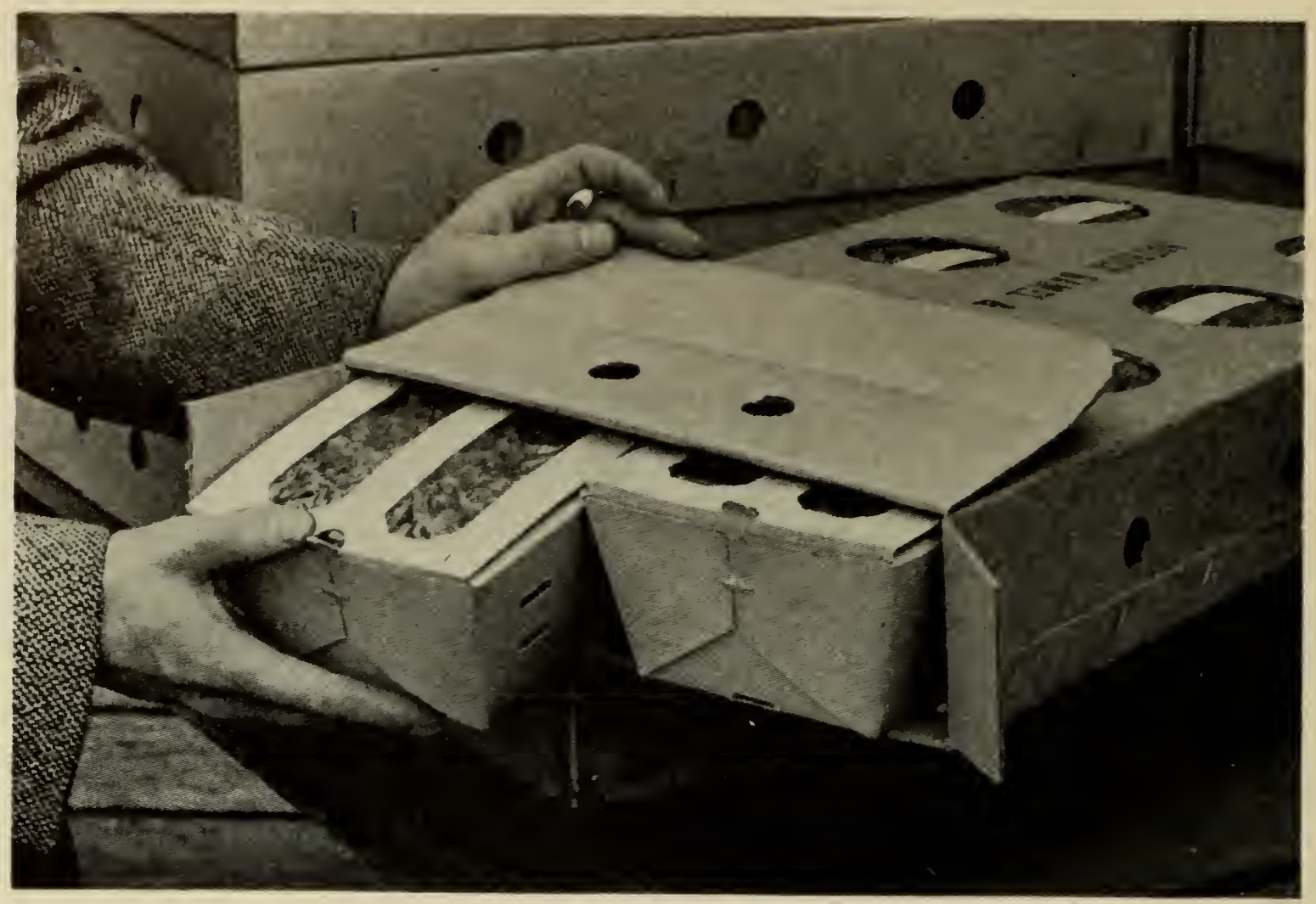

Fig. 5.-Emballage parfait pour le marché de détail. Cette boîte contient 8 casseaux de carton (1 pinte) avec couvercle à fenêtres de cellophane.

(Courtoisie du "Bulletin des Agriculteurs").

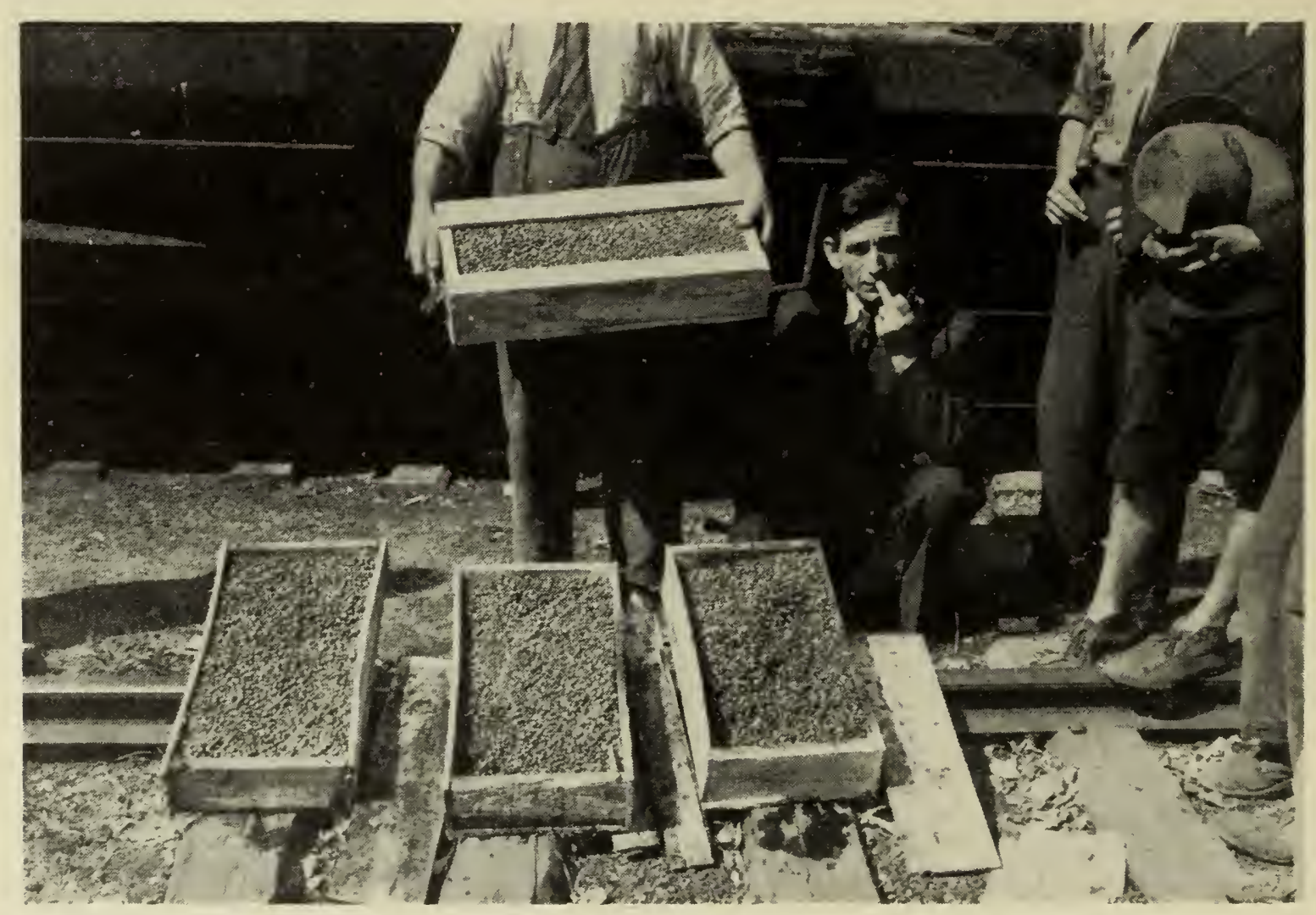

Fig. 6.- Sur le sol, boites rejetées à l'inspection. Remarquer la paurre qualité du bois et la mauvaise confection. Ces boîtes ne sont pas assez remplies. A comparer avec celle que tient l'inspecteur.

(Courtoisie du "Family Herald and Weekly Star"). 
Le district d'Abitibi-'Témiscamingue expédic toute sa production en paniers de 11 pintes, tandis qu'au Lac St-Jean, on emploie exclusivement la boîte de 22 livres pour le marché d'exportation. La boîte ordinaire est fabriquée dans les moulins avec du bois d'épinette non plané et est assemblée par le cueilleur luimême, dans la bluetière. La qualité et l'apparence de ces boîtes laissent généralement à désirer. (Fig. 6). Cet emballage grossier nuit certainement à la popularité du bluet du Lac St-Jean sur les marchés. Des acheteurs progressifs ont entrepris, en ces derniers temps, de réemballer les bluets avant l'expédition. Les boîtes ordinaires reçues du champ sont dirigées à un poste central, vidées et mises de côté. Les bluets sont nettoyés et remis dans des boîtes plus attrayantes faites de bois plané. Certains recouvrent même l'intérieur de ces boîtes d'une enveloppe de papier. (Fig. 7).

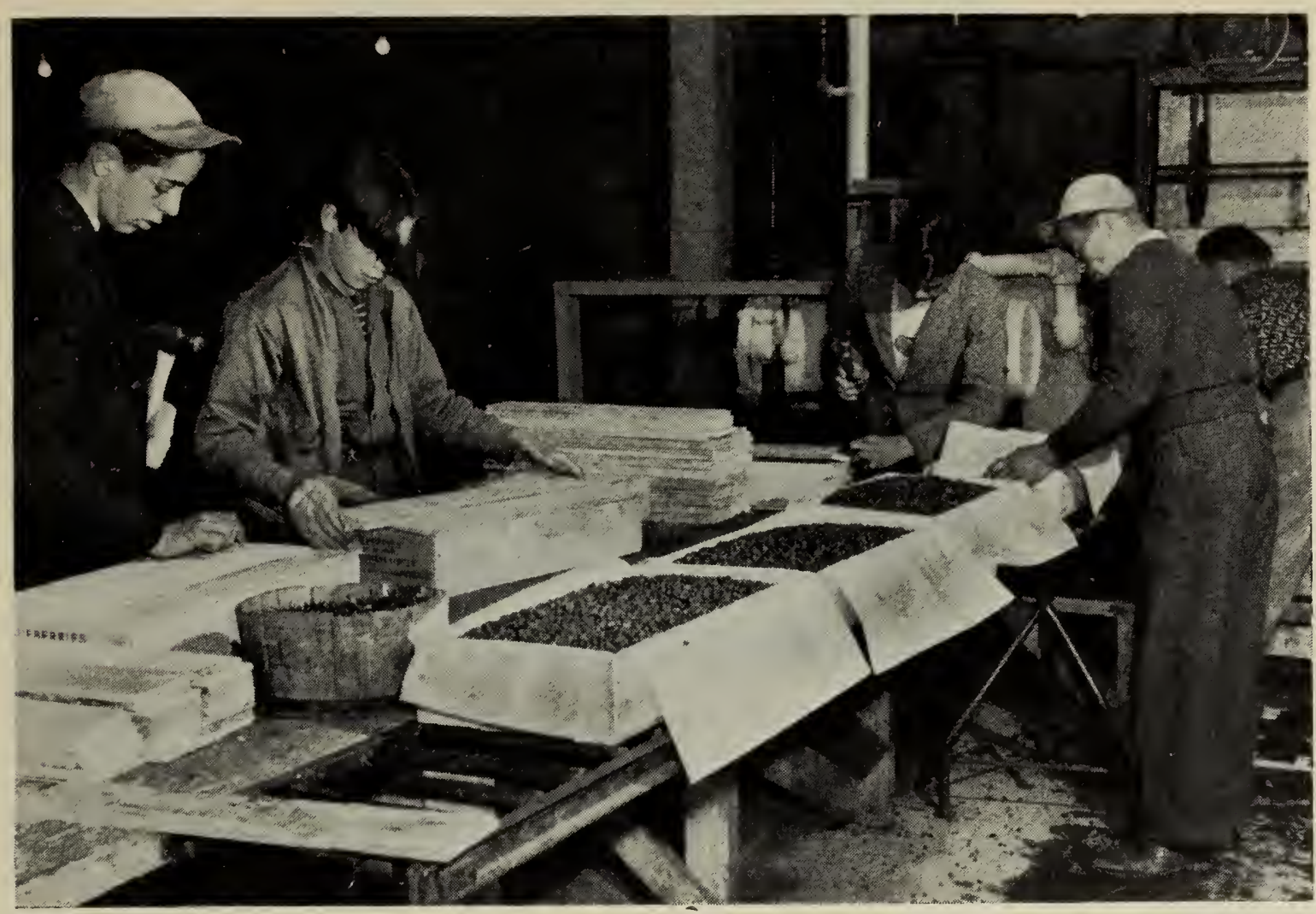

Fig. 7.--Réemballage des bluets en boîtes de bois plané avec doublure de papier eiré, par Canada Packers Ltd, à Dolbeau, Lac St-Jean.

(Courtoisie du "Bulletin des Agriculteurs").

\section{Classification et inspection.}

La classification et l'inspection des bluets relèvent du ministère provincial do l'Agriculture. (Fig. 8). Dans Québec, les cueilleurs ne peuvent mettre en vente, et les acheteurs n'expédier, que les bluets appartenant aux catégories suivantes et dûment identifiés, selon le règlement précité:

"Catégorie no 1: bluets sains, bien conformés, d'un beau coloris, assez uniformes, d'au moins un quart de pouce de diamètre, sans queue, ni feuilles, ni fruits verts, ni terre ni autre matière étrangère.

"Catégorie no 2: bluets sains, assez bien colorés, sans dégâts sérieux causés par la terre ou autres matières étrangères, ou par quelque autre cause."

En pratique, pas un des expéditeurs ne veut accepter les bluets de la seconde catégorie. Seuls quelques fabricants de conserves en prennent des quantités très limitées.

L'application des règlements de vente a donné de bons résultats. Les boîtes utilisées sont de fabrication plus soignées et de dimensions assez uniformes. Quant 
à la qualité des bluets, il y a encore beaucoup à faire pour l'améliorer et l'on compte sur la probité des cueilleurs et des expéditeurs qui n'ont pas toujours bien compris leurs véritables intérêts.

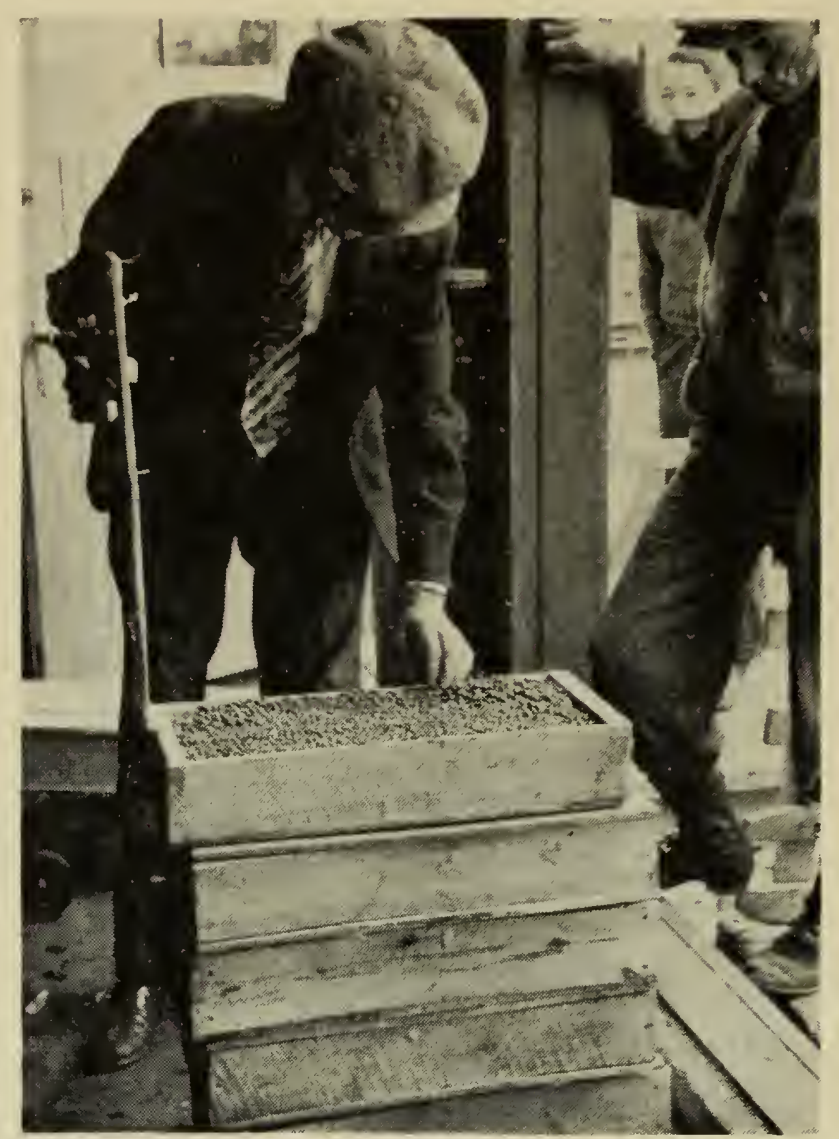

Fig. 8. Inspection des bluets au point d'expédition (Lac St-Jean). Boîte ordinaire, de confection conforme aux règlements, et bien remplie de fruits de bonne qualité.

(Courtoisie du "Bulletin des Agriculteurs").

\section{Amélioration de la qualité des bluets.}

II est vrai que la cueillette et l'expédition des bluets se font dans des conditions où il est difficile de ne pas endommager les fruits. Les points d'expédition étant souvent fort éloignés des centres de cueillette, les bluets doivent être transportés en camion sur des routes forestières souvent rudimentaires. Et du point d'expédition au lointain marché, ils ont à supporter plusieurs jours de transport par chemin de fer. Cela entraîne de graves inconvénients pour le commeree de ce fruit périssable à un degré élevé.

Selon la "Food and Drug Administration" des Etats-Unis, les quantités de bluets frais du Québec rejetées sont, chaque année, considérables. Par exemple, en 19.4, elles se sont élevées à 600,000 livres; en 1945, à 525,000 livies, et en 1946, à 32,000 livres. Citant ces chiffres, Johns (10) rapporte que la cause du rejet de ces bluets est le taux élevé des moisissures révélé par l'analyse microscopique (moississures invisibles à l'oeil nu) plutôt que la présence du ver du bluet. Les bluets du Québec sont heureusement exempts de ce ver, au contraire de ceux du Maine et des Provinces Maritimes.

Cette observation appelait l'étude du développement de ces moisissures. Un premier examen au moment de l'expédition a montré que le pourcentage des moisissures dans les boîtes de bluets sains, fermes, etc. (catégorie no 1), est alor's 
pratiquement le même que daus les boîtes de bluets rejetés comme "mouillés", trop mûrs ou écrasés. C'est au cours du transport vers le marché que les moisissures semblent se développer sur le jus et la pulpe des fruits écrasés ou brisés. Plus il y a de tels fruits, plus le décompte des moisissures est élevé.

D'autres épreuves ont été faites pour l'étude de l'influence de la température sur la multiplication des moisissures. Une boîte de bluets du Lac St-Jean fut apportée à Ottawa, par automobile et par train. De ces bluets on a fait trois lots: (a) bluets choisis pour leur fermeté; (b) bluets écrasés ou endommagés autrement; (c) bluets non sélectionnés, tels que trouvés dans la boîte. Un casseau de chaque catégorie fut placé à chacune des températures suivantes, 40, 60 et 72 degrés F. On en fit ensuite l'analyse, à intervalles divers. Les résultats obtenus paraissent dans le tableau V.

\section{'TABLEAU V}

\section{DÉVELOPPEMENT DES MOISISSURES SUR BLUETS DU LAC ST-JEAN (10)}

(Traitements commencés 72 heures après la cueillette)

\begin{tabular}{|c|c|c|c|c|c|c|c|c|c|}
\hline \multicolumn{10}{|c|}{ Pourcentage des champs positifs (moisissures) (méthode Howard) } \\
\hline \multirow{3}{*}{$\begin{array}{l}\text { Durée de } \\
\text { l'entreposage } \\
\text { (jours) }\end{array}$} & \multicolumn{9}{|c|}{ Température d'entreposage } \\
\hline & \multicolumn{3}{|c|}{$40^{\circ} \mathrm{F}$. } & \multicolumn{3}{|c|}{$60^{\circ} \mathrm{F}$. } & \multicolumn{3}{|c|}{$72^{\circ} \mathrm{F}$} \\
\hline & $\Lambda$ & B & C & $\Lambda$ & B & $\mathrm{C}$ & $\Lambda$ & $B$ & $\mathrm{C}$ \\
\hline $\begin{array}{r}0 \\
1 \\
4 \\
6 \\
6 \\
8 \\
10\end{array}$ & $\begin{array}{l}4 \\
2 \\
2 \\
2\end{array}$ & $\begin{array}{l}20 \\
16 \\
21 \\
43\end{array}$ & $\begin{array}{l}6 \\
2 \\
4 \\
9\end{array}$ & $\begin{array}{r}2 \\
4 \\
18 \\
18 \\
31\end{array}$ & $\begin{array}{l}17 \\
25 \\
53 \\
4.1 \\
84\end{array}$ & $\begin{array}{r}6 \\
12 \\
34 \\
24 \\
71\end{array}$ & $\begin{array}{r}4 \\
3 \\
4 \\
14 \\
36 \\
80\end{array}$ & $\begin{array}{l}23 \\
38 \\
38^{\prime \prime} \\
55^{\prime \prime} \\
47^{\prime \prime} \\
96^{\prime \prime}\end{array}$ & $\begin{array}{r}5 \\
10 \\
25 \\
25 \\
24 \\
79^{\prime \prime}\end{array}$ \\
\hline \multicolumn{4}{|c|}{$\begin{array}{l}\text { A-Bluets choisis, fermes. } \\
\text { C-Bluets non choisis. }\end{array}$} & \multicolumn{5}{|c|}{$\begin{array}{l}\text { B--Bluets écrasés. } \\
\text { (") Forte odeur de moisi. }\end{array}$} & \\
\hline
\end{tabular}

"Ces réeultats, dit Johns (10), suggèrent que les bluets fermes ne sont pas susceptibles de donner de forts décomptes de moisissures, même après avoir été tenus à la température de la chambre pendant plus d'une semaine. D'un autre côté, les bluets endommagés, dans lesquels il se peut que des moisissures se soient développées avant leur arrivée à Ottawa, montrèrent au début un faible décompte. Mais après une certaine exposition à la température de la chambre, le pourcentage s'éleva, et l'on pouvait sentir une forte odeur de fermentation. Si, cependant, les bluets endommagés étaient tenus à 40 degrés, le décompte ne s'élevait guère avant la huitième journée de l'épreuve. Ces résultats démontrent que l'inconvénient des moisissures microscopiques (invisibles) peut être évité par un refroidissement rapide des bluets à 40 degrés et l'observance de cette température jusqu'à destination."

A la lumière de ces observations, bien qu'elles soient forcément préliminaires, on peut faire certaines recommandations utiles aux expéditeurs el aux cueilleur's. 


\section{Récommandations}

Aux expéditeurs. Le meilleur moyen d'éviter les pertes dues aux moisissures serait, sans doute, la congélation ou au moins le pré-refroidissement avant l'expédition. Le pré-refroidissement, qui a été mis à l'essai en ces dernières années, et avec assez de facilité au point de vue technique, comprend trois opérations: (a) nettoyage des bluets à la réception; (b) refroisissement au moyen d'un courant d'air à 40 degrés F.; (c) empaquetage en boîtes de choix (bois plané, avec ou sans revêtement intérieur de papier ciré). Ces bluets sont immédiatement chargés à bord de wagons réfrigérés, et expédiés.

A défaut de cette première manière de procéder, il est recommandé aux expéditeurs de veiller à ce que les bluets obtenus des cueilleurs soient de la meilleure qualité possible et d'en surveiller le transport par camion et la manipulation au point de chargement. Ils doivent aussi s'assurer que le "glaçage" des wagons soit suffisant et fait à temps, éviter de surcharger, et disposer les boîtes de sorte que la circulation de l'air froid se fasse aussi abondante que possible. Voici, sur se sujet, les recommandations faites par les Chemins de fer Nationaux du Canada.

Choix et préparation des wagons. On doit se servir de wagons propres, secs et exempts de mauvaises odeurs. L'expéditeur doit commander à l'avance un nombre suffisant de wagons afin qu'on ait le temps de les mettre en bonne condition et de les bien refroidir. Voir à ce que le système de refroidissement fonctionne bien dans toutes ses parties et que le wagon soit déjà refroidi au moment du chargement.

\section{Chargement des wagons.}

1. La capacité d'un wagon réfrigéré régulier canadien est de 1584 boîtes, soit 33 piles de 48 boîtes (12 boîtes en hauteur et 4 boîtes en largeur, en travers du wagon).

2. On pile les boîtes en partant des murs du bout. Il y aura 17 piles d'une extrémité du wagon au centre, et 16 piles de l'autre extrémité vers le centre.

3. La première pile doit toucher le mur du bout du wagon. Les boîtes sont placées sur le plat, quatre par quatre, en travers du wagon. On laissera un espace égal entre les boîtes et les murs de côté et entre chaque boîte. (Voir fig. 9). La pile ordinaire comprend 12 boîtes superposées. La deuxième pile touchera à la première en s'y appuyant fermement, et ainsi de suite. Les boîtes se suivront bien en ligne d'une pile à l'autre. Autant que possible, compléter les piles.

4. Mettre des lattes en travers des boîtes sur la rangée inférieure, sur la rangée supérieure, de même qu'entre toutes les deux rangées. (Voir figure 9). L'une des lattes touche le mur de gauche et l'autre, celui de droite. Les lattes mesurent 8 pi. $x 1$ po. $x \quad \frac{1}{2}$ po. Elles sont clouées aux extrémités de chaque boîte avec du clou de $11 / 4$ po.

5. Au centre du wagon, les piles ne se touchent pas. Il reste un espace de 2 pi. 2 po. à combler de façon à consolider toute la charge. On bâtit un échafaudage selon le plan donné à la figure 10. Si le wagon n'est pas rempli à sa capacité, on doit quand même consolider les piles de boîtes.

Les mêmes principes s'appliquent aux wagons irréguliers pourvu qu'on tienne compte de leurs dimensions.

Avantages de cette méthode. Cette méthode est de nature à favoriser (a) une plus grande résistance des murs du bout du wagon, (b) une meilleure circulation d'air à travers toute la charge, (c) une inspection plus facile en cours de route, (d) un minimum de dommages aux boîtes soumises à des secousses inévitables aux départs et aux arrêts. 


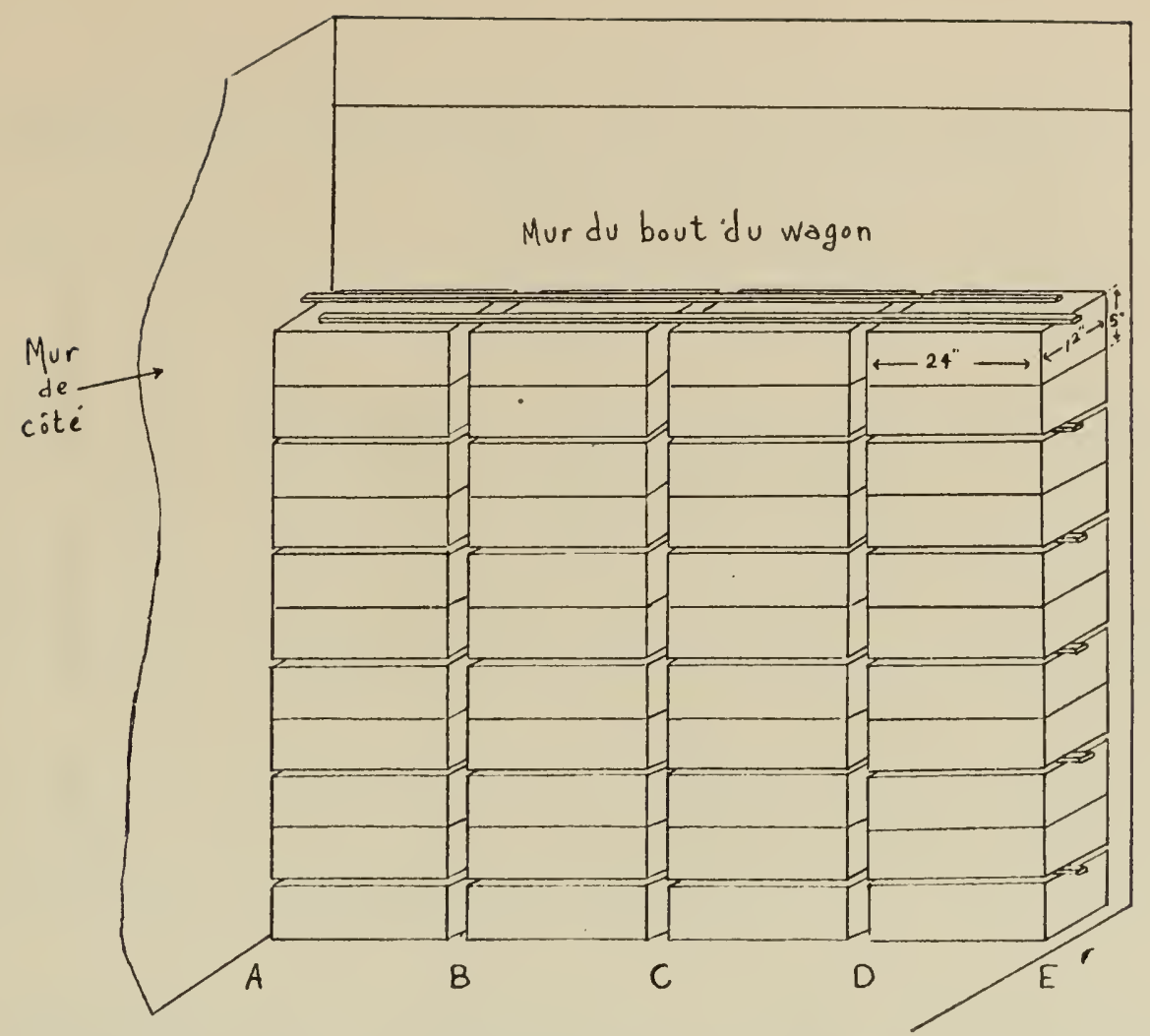

Fig. 9.-Manière de disposer les boîtes de bluets en pile dans un wagon régulier du C.N.R.

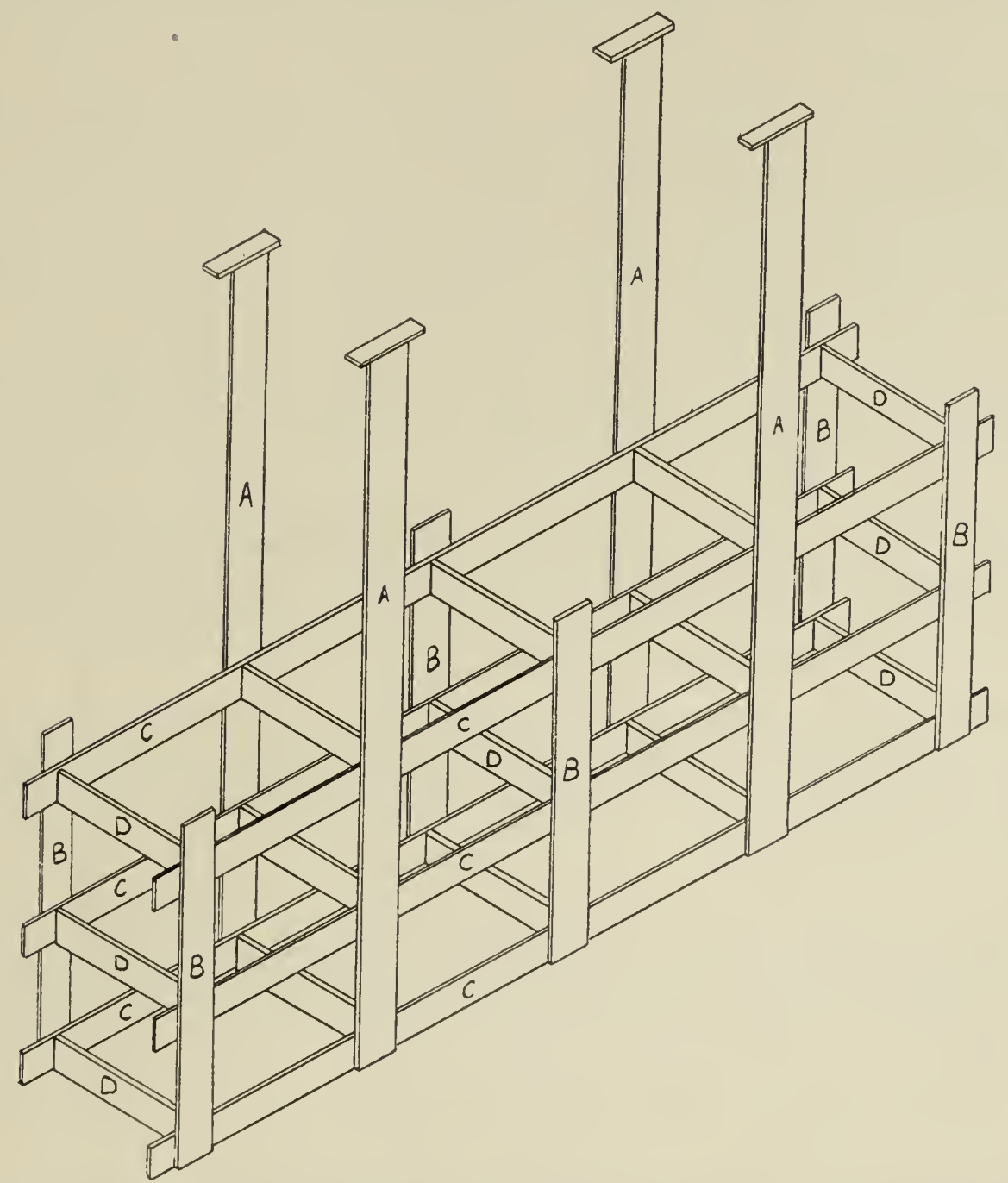

Fig. 10.-Echafaudage placé en travers du wagon, au centre, pour consolider la charge de boîtes de bluets. 
A. Montants rejoignant le plafond du wagon, bois de 2 po. $x 6$ po.

B. Montants en bois de 2 po. $x 6$ po. dont la hauteur doit être la hauteur actuelle des piles de boîtes. On les place à distances égales correspondant aux lettres A, B, C, D et E de la figure 9 (droite).

C. Traverses en bois de 1 po. $x 4$ po. $x 8$ pi. 4 po. (la largeur d'un wagon régulier du C.P.R. ou C.N.R. est de 8 pi. 4 po.).

D. Traverses en bois de 2 po. $\times 4$ po., trois pour chaque montant $B$.

Aux camionneurs. Le camionneur est un intermédiaire important. De la bluelière au point d'expédition, c'est lui qui est responsable de la qualité des bluets. S'il fait lui-même l'achat des bluets, il doit veiller à ne prendre que des bluets satisfaisant aux exigences de la loi. En cours de route, il conduira sa voiture de façon à éviter de trop dures secousses. Il fera livraison des bluets dans le plus court délai possible. Il aura soin de couvrir les boîtes pour qu'elles ne s'échauffent pas au grand soleil, qu'elles ne prennent pas trop la poussière ou ne soient pas exposées à la pluie.

Aux cueilleurs. II importe que le cueilleur n'offre en vente que des bluets de première qualité. Il doit étudier et respecter les règlements concernant la qualité des bluets et les emballages. Il lui faut attendre, pour cueillir le bluet, que ce dernier soit en pleine maturité. Le bluet mûr est ferme, entièrement recouvert de sa "poussière bleue" (pruine) qui lui donne tout son attrait, et qui surtout, de par sa nature cireuse, bouche les pores de la peau, abaisse la transpiration au minimum, et permet au fruit de se conserver plus facilement et plus longtemps. De plus, le bluet mûr se détache plus facilement de la grappe, et donc il est plus agréable et plus "d'avance" à cueillir. Au contraire, le bluet non encore mûri est rougeâtre et très acide. S'il y a une certaine proportion de fruits verts ou à moitié mûrs dans les boîtes, ils feront "mouiller" tout le contenu qui ne tardera guère à développer des moisissures. Il est difficile de faire disparaître les bluets verts par le criblage ordinaire. Enfin, on ne doit pas cueillir le bluet lorsqu'il est humide de rosée ou de pluie, parce qu'alors il se conserve très mal.

Le cueilleur doit manipuler les boîtes avec la plus grande précaution. Les boîtes seront remplies de bluets à leur pleine capacité. Il est recommandé de les emplir par le côté, en donnant des secousses légères et répétées. Si la masse des bluets est compacte, elle obéira moins aux heurts inévitables pendant le transport par camion, bateau ou chemin de fer: c'est en glissant les uns sur les autres que les bluets perdent leur pruine et ont tendance à "mouiller".

Il est sage aussi de ne pas emballer les bluets dès l'arrivée du champ. La température des fruits peut facilement atteindre 90 degrés $\mathrm{F}$. dans les récipients servant à la cueillette par les journées très chaudes. Il vaudrait mieux les laisser perdre un peu de cette chaleur au cours de la nuit suivante avant de les mettre dans les boîtes. Ces boîtes seront espacées entre elles et placées à l'ombre.

Le ceuilleur ne doit pas retenir des quantités de bluets pendant plusieurs jours avant de les vendre dans l'espérance d'une hausse de prix. C'est un mauvais calcul. Ces bluets veillis ont toutes les chances d'être refusés, rendus à destination, et d'indisposer le client étranger.

Il reste bien établi que l'amélioration de cette production est irréalisable sans la bienveillante coopération des cueilleurs, d'ailleurs principaux intéressés. Ils savent par expérience que le marché des bluets est le plus instable qui soit et doivent comprendre qu'on ne saurait réussir à le stabiliser sans leur collaboration à ce premier stage. 


\section{PARTIE IV}

\section{GULTURE DE LÁ BLUETIÈRE}

Il faut se rendre compte que l'expression "culture du bluet sauvage" n'implique pas, comme dans le cas du bluet géant amélioré ou d'une variété quelconque de petit fruit, la multiplication des plants en serre ou en couche, ni l'aménagement, l'amendement et la fertilisation d'un terrain déterminé, ni la taille et la protection contre les maladies et les insectes par les méthodes ordinaires. La culture d'une bluetière naturelle comporte plutôt les opérations nécessaires pour augmenter le rendement, si possible, et améliorer la qualité du fruit.

Cette culture pourrait être pratiquée avec avantage par les cultivateurs ou colons possédant une certaine étendue de "terre à bluets" dont ils ne sauraient tirer plus de revenu avec les récoltes ordinaires, grains, foin, etc. Elle serait praticable encore sur des terrains détachés par voie de concession des Terres de la Couronne.

On n'a pas, sur le sujet de la culture des bluetières, toute l'information qu'on voudrait. Les recherches ont été relativement peu nombreuses, aux Etats-Unis comme au Canada. Mais avant de passer en revue les quelques résultats obtenus, il convient de considérer quelques difficultés inhérentes qui rendent les recherches difficiles.

Origine et durée d'une bluetière. Une bluetière est chose plus compliquée qu'il ne paraît à première vue. Elle ne représente rien de permanent, mais plutôt un état transitoire de la forêt en croissance. Toutes les forces de la nature qui s'y exercent tendent à rétablir les essences forestières adaptées au sol et au climat. Maintenir une bluetière en état de production, la "cultiver", est donc une lutte constante contre ces forces qu'il faut connaître. La recherche a, jusqu'à date, porté sur les facteurs naturels qui entrent en jeu.

Plusieurs se demandent comment il se fait qu'après l'incendie d'une forêt oì l'on ne voyait que peu ou pas de bluets du tout il y naisse tout-à-coup une végétation serrée de plantes à pousse basse, dont le bluet. Le phénomène illustre bien le fait que, dans le règne végétal, les plantes s'associent et se succèdent par groupes, selon les conditions de terrain et de climat.

Qu'on imagine une épaisse forêt du nord du Québec comprenant des r'ésineux (épinette noire, pin gris ou cyprès ou des arbes feuillus (tremble, peuplier, bouleau). (Voir le graphique I). En fouillant le sol en surface, on trouverait, en plus des racines des grands arbres, un enchevêtrement de tiges souterraines ou de rhizomes et de racines qui ne peuvent pas pousser-ou très peu-de tiges aériennes viables tant que certaines conditions différentes ne s'établiront pas: modification sensible de l'acidité du sol et plus grande abondance de lumière. Mais voici que la hache, du bûcheron ou bien le feu viennent éclaircir la végétation forestière. II est rare 

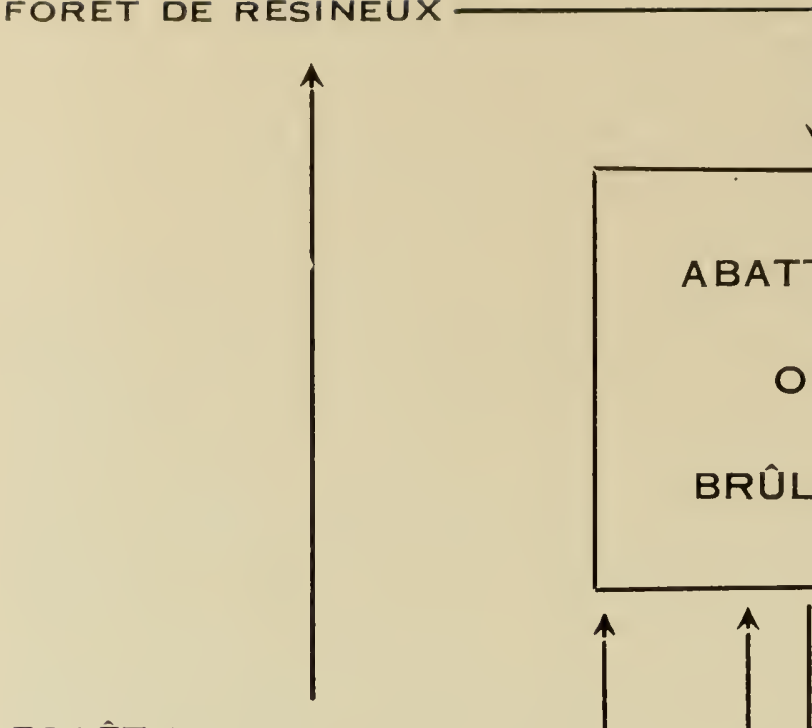

FORETT DE FEUILLUS

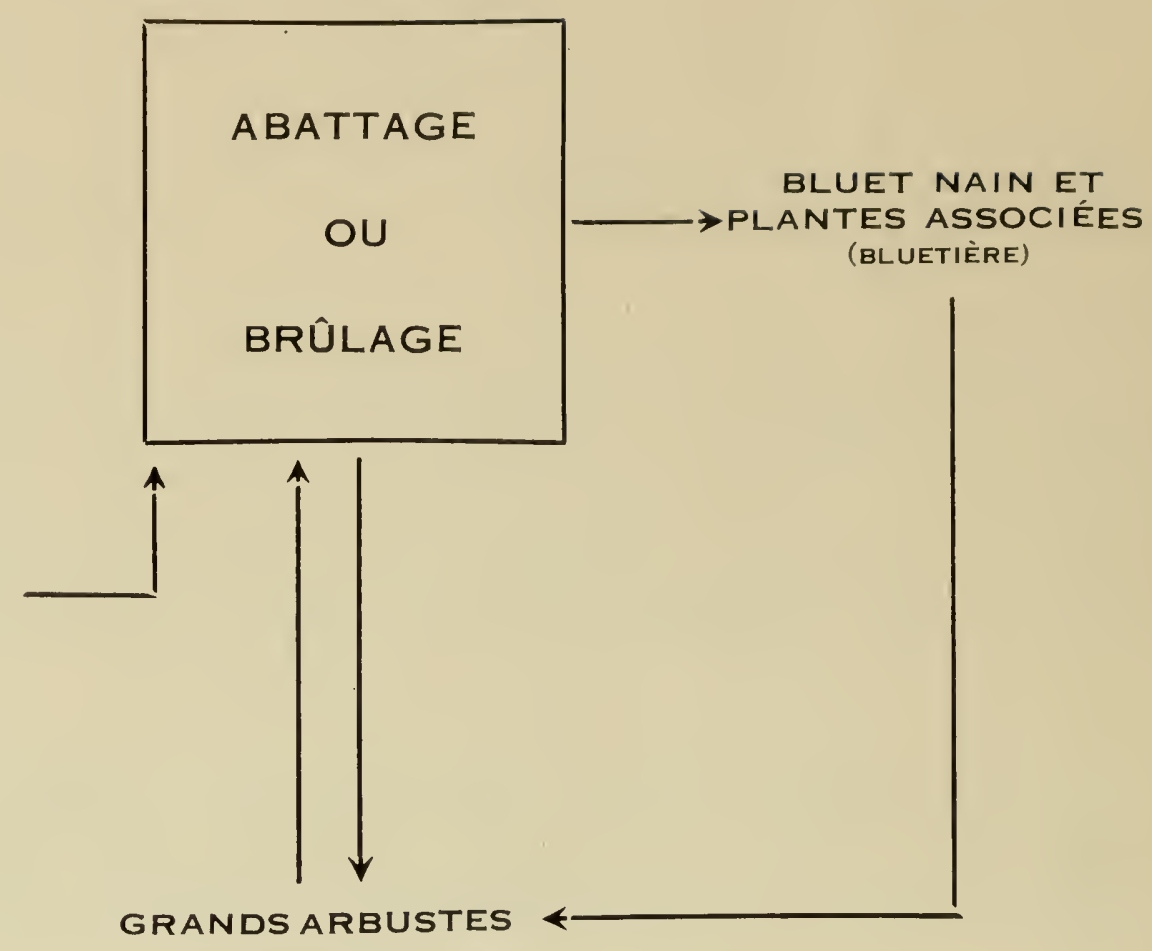

BLUETS GÉANTS ET

PLANTES ASSOCIÉES

(BLUETIĖRE)

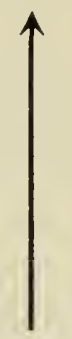

GRÈVE SABLONNEUSE

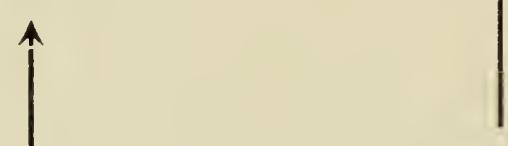

MÉLÈZE ET

PLANTES ASSOCIÉES

MERÉCAGE

Graphique I.-Cycle permettant de situer la bluetière dans l'évolution des terrains acides. La partie supérieure représente ce qui se passe dans l'habital du bluet nain sauvage. La partie inférieure représente l'habitat du bluet géant sauvage.

que le feu brûle assez profondément pour atteindre les racines et les rhizomes. Ici et là vont sourdre, l'année suivante, des tiges de bluets et d'autres plantes, presque toujours les mêmes: comptonie ou bois de savane, kalmie ou crevard de moutons, fougères, petit thé, apocyn, etc. (Fig. 11). 


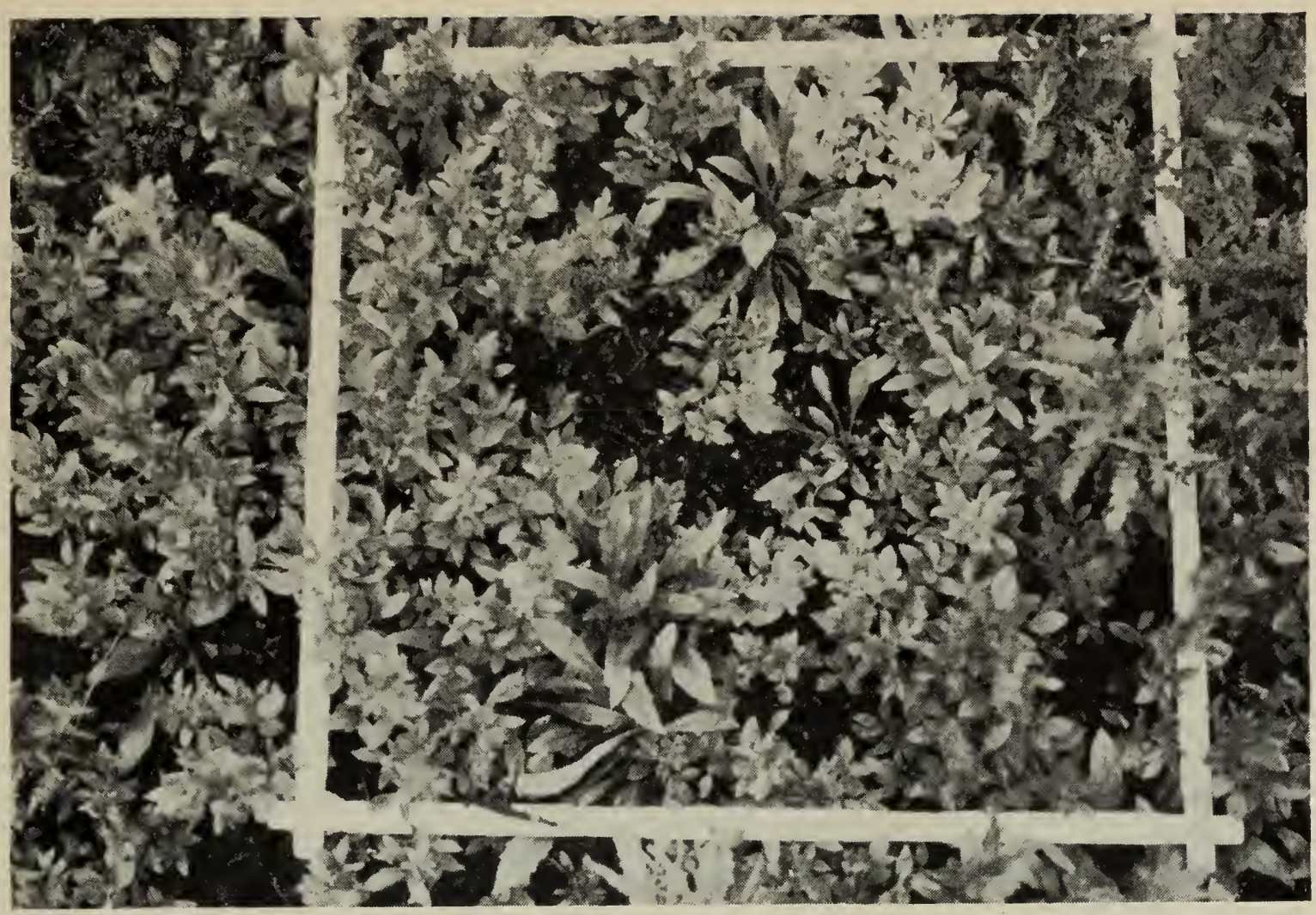

Fig. 11.-Jeune peuplement de bluets et plantes associées, photographié le 14 juillet, sur un terrain brûlé au printemps de la même année. Un oeil exercé distinguera au moins trois espèces différentes.

En même temps, les grandes aulnes s'installent avec les rejetons du peuplier, du tremble et du bouleau, pendant que les conifères (épinette, cyprès) germent ici el là. Pour quelques années, la récolte des bluets y est intéressante. Mais quand la bluetière prend de l'âge, les arbustes croissent et portent ombrage au bluet. Bientôt le bluet disparaî̀, comme les autres plantes basses, étouflé par la grande végétation. Dans quelques années, le bosquet sera redevenu forêt. (12).

Ce phénomène naturel peut-il être interrompu à un moment donné, de facon à maintenir la bluetière en production? Oui, par un ensemble de moyens pratiques de culture qui sont le brûlage, le désherbage, le contrôle des maladies et insectes, et la fertilisation du sol.

\section{BRÛLAGE}

Le bluet nain ne diffère pas des autres plantes à fruits. Il a besoin, pour produire abondamment et régulièrement, qu'on le taille, qu'on lui fasse de l'espace et que, parfois, on supplée au manque de nourriture.

Effet sur le rendement de la bluetière. Les cueilleurs de bluets ont adopté le brûlage périodique comme moyen de renouveler la bluetière. C'est, en réalité, afin de tailler les plants. Le feu, s'il est mis au moment voulu, détruit les vieilles tiges aériennes du plant, mais n'endommage pas les tiges souterraines ou rhizomes. Au cours de la saison de végétation suivante, de nouvelles tiges sortent des bourgeons localisés sur les rhizomes et croissent intensément (Fig. 12), el sur chacune se forment des bourgeons à fruits. Il pousse en moyenne deux tiges nouvelles pour chaque vieille tige brûlée. Si le plant avait été fauché seulement à une certaine distance 


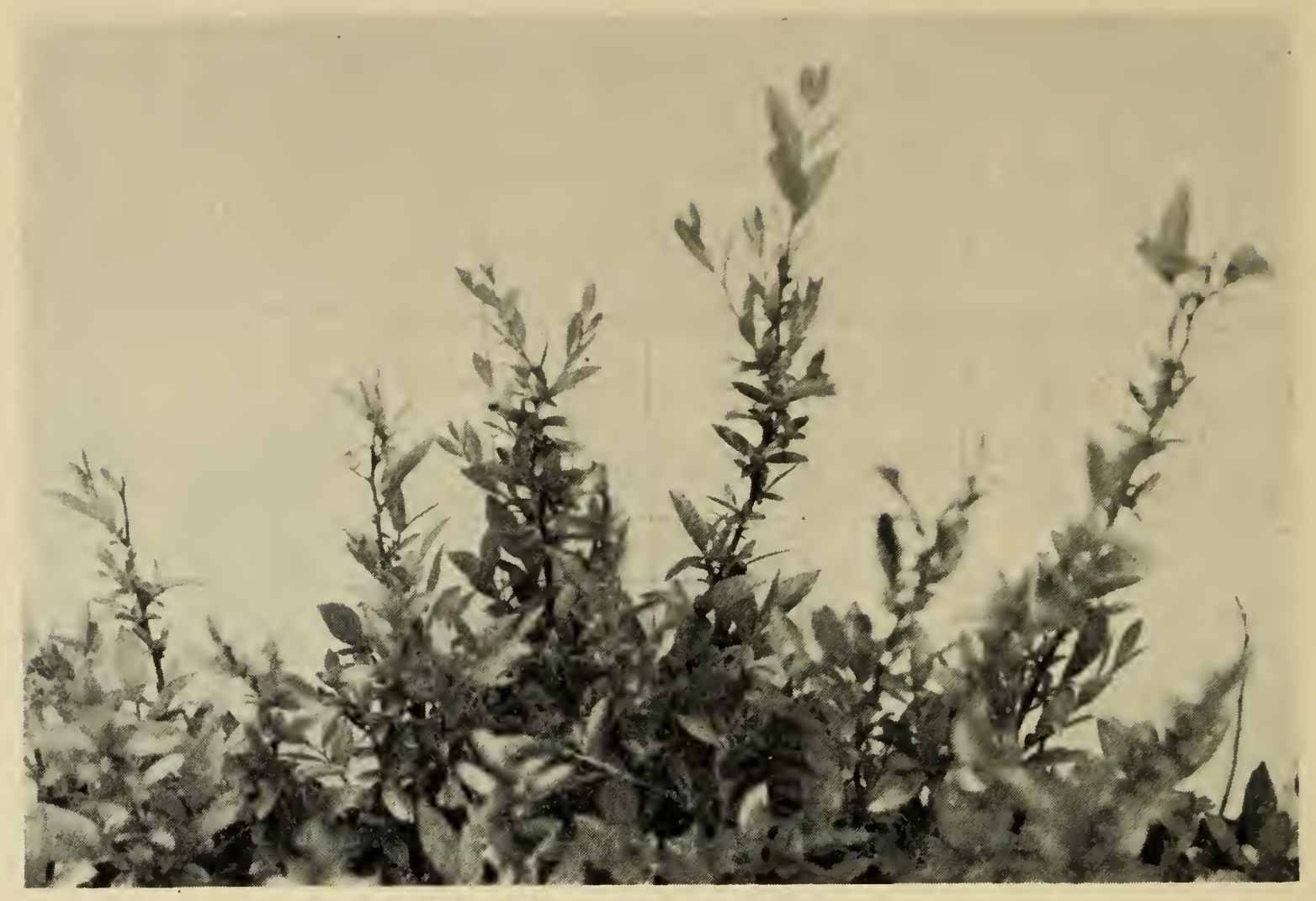

Fig. 12.-Deuxième saison de végétation après un brûlage de printemps, contre un quadrillé à l'échelle de 1 pouce. Brûlage au printemps 1938, photographié le 14 juillet 1939. (Lac St-Jean).

du sol, de nouvelles tiges proviendraient aussi des bourgeons laissés à la lige aériemne amputée.

L'année suivante, les bourgeons à fruits fleurissent et produisent une forte récolte de fruits, la meilleure. Les récoltes suivantes seront de moins en moins abondantes. Chandler et Mason (3) ont observé que lorsque le $V$. pennsylvanicum est l'espèce prédominante dans la bluetière, la première récolte après la taille est de beaucoup meilleure que les récoltes subséquentes. Mais là où c'est le $V$. canadense qui l'emporte, la deuxième récolte surpasse la première. Cela s'entend pour les bluetières du Maine. Dans le Québec, du moins au Lac St-Jean, où les deux espèces cohabitent étroitement, cette différence n'a pu être observée.

Pratiques populaires. Dans le Québec, peu de cueilleurs de bluets, šil en est, ont adopté le brûlage périodique comme moyen de culture, pour une bonne raison, parce que les champs où ils cueillent les bluets sont des terrains appartenant à la Couronne ou des limites concédées aux compagnies forestières. Dans les Provinces Maritimes où, depuis plusieurs années, de grandes étendues de terrains à bluets appartiennent à des sociétés, cette méthode d'exploitation s'est offerte logiquement. En Nouvelle-Ecosse et au Nouveau-Brunswick, selon Eaton (9), on prend un soin systématique des bluetières: l'étendue est divisée en trois parties d'égale superficie, et, chaque année, une partie est brûlée, tandis qu'on récolte les deux autres. La rotation est donc de trois ans. On fait de même dans l'Etat du Maine. (3)

Certains producteurs ont l'habitude de faucher toute la végétation de la bluetière avant le brûlage, aussi tard que possible à l'automne. D'autres se contentent de faucher les seuls endroits où abondent les plantes étrangères, ou même seulement ces dernières sans couper les bluets, s'ils en ont les loisirs au cours de la saison. Ce matériel sèche sur place et permet un feu nourri. 
Dans les bluetières où la végétation est dairsemée, il devient nécessairte d'ajouter un combustible sous forme de foin ou de paille. C'est une pratique courante dans l'Etat du Maine. Chandler (2) recommande qu'on étende le combustible aussi uniformément que possible, à raison d'une demie à une tonne à l'acre selon la densité de la végétation ordinaire. La paille ou tout autre débris végétal est préférable au foin, parce que ce dernier est susceptible d'introduire dans la bluetière des mauvaises herbes comme les trèfles, chiendent, verge-d'or, pissenlit et épervière. La même recommandation est faite dans les Provinces Maritimes.

Certains producteur's, enfin, répandent de l'huile ou de l'essence (gazoline) sur le terrain, d'autres se servent de torches à essence. Ces pratiques sont coûteuses et comportent certains dangers.

On manque de chiffres pour établir une comparaison définitive dans le coût de l'emploi de ces divers combustibles.

A l'automne ou au printemps?-Le brîlage peut se faire en deux saisons, à l'aulomne avancé ou de bonne heure au printemps. Laquelle est préférable?

Voici l'opinion de Chandler (2) sur le brûlage d'automne: "En général, les producteurs ne songent pas au brûlage d'automne, mais cette pratique devrait être en usage pour certains sols. Le brûlage d'automne comporte quatre avantages: (a) il aide à tenir en échec le crevard de moutons (kalmia); b) on peut le maîtriser plus facilement; c) il ne détruit pas les nids de bécasses (faune du Maine); d) il peut aider les producteurs à faire un emploi plus économique de leur main-d'oeuvre. Pour le brûlage d'automne, on peut utiliser du foin, mais il en faudra probablement plus que pour un brûlage de printemps. Certains producteurs trouveront moins dispendieux d'employer de l'huile que du foin. Le brûlage d'automne ne devrait pas être commencé tant que les feuilles ne sont pas tombées.

Fréquence du brûlage.-Une période de trois ans est-elle trop longue ou trop courte? L'expérimentation n'a pu encore en décider nettement.

Pour ce qui est du plant de bluet lui-même, on a établi (a) que le brûlage trop fréquent n'augmente pas la densité des plants (4) et (b) que, sur une longue période, c'est avec le brûlage triennal qu'on tient au maximum le rendement de la bluetière, les autres conditions étant normales (3). Par exception, dans le cas d'un fléau de vers du bluet - inconmus dans Québec-, certains producteurs n'hésitent pas à perdre une récolte de bluets pour brûler de nouveau deux ans après un premier brûlage.

Au point de vue du sol, le brûlage, fréquent ou rare, doit être considéré comme IIII mal nécessaire. Il a de sérieux inconvénients. Chandler (2) dit que le brûlage "empêche l'accumulation de la matière organique, détruit l'azote et transporte le phospore à la surface du sol où il se fixe à des composés que la plante ne peut assimiler". L'accumulation de la matière organique est importante, parce qu'elle aide à conserver l'humidité du sol, permet la croissance de plants plus vigoureux et la cueillette de fruits plus gros. L’intervalle entre les brûlages, dans un sol paurre ou dépourvu de matière organique, ne sera donc jamais trop long.

Pour ce qui regarde la province de Québec, il ne semble pas encore possible de conseiller une seule et unique longueur de rotation pour tous les terrains à bluets. Plus la couche d'humus dans le sol est mince, plus on devra espacer les brûlages et mettre de soin à attendre les conditions idéales pour brûler.

Recommandations générales.-La clef du succès dans l'opération du brûlage c'est la rapidité et l'uniformité. Le brûlage est d'autant plus efficace que les débris végétaux en surface sont secs et que le feu ccurt rapidement aidé d'une brise légère. Plus le brûlage sera uniforme, meilleur sera le rendement de la bluetière, et surtout meilleur sera le contrôle des insectes, là où ce fléau exerce ses ravages. On a observé, 
('n effel (9), que le ver du blued mel plus de lemps à se réinstaller dans une bluetière bien brêlée que dans celle oì on lui a laissé des "ilots de résistance". II faut done, avant de melle le feu, šassurer qu'il y a suffisamment de matériel à brûler.

Au printemps, le brîlage doil êlre lail de bomme heure des que la fonte des nejeges est finie. Si le champ est irrégulier el comporte des endroits où la neige séjourne plus longtemps, on lera le brûlage à des périodes dillérentes, en brûlant rhaque section aussitôt qu'elle sera assez sèrhe. Cela dans le but de protéger l'humus du sol.

Linfin, on doit user d'une prudence extrême lors du brûlage et garder toujours la maîtrise du feu. Cette période est celle où l“indice d'inflammabilité des forêts est le plus élevé. Une seule imprudence peut amener un désastre.

II est fortement recommandé aux producteurs de demander l'aide d'un gardefeu pour procéder au brûlage d'étendues considérables. Celui-ci les assistera de ses connaissances techniques. Il est en mesure de juger de l'état du sol et des conditions de l'opération. Il utilisera comme coupe-feu les clairières naturelles ou ordonnera l'abattage des arbres et arbustes sur une largeur d'au moins 25 pieds pour arrêter la marche du feu à l'endroit désiré et protéger la forêt ou la brousse environnante. II tiendra compte de la vitesse et de la direction du vent et les emploiera avec avantage.

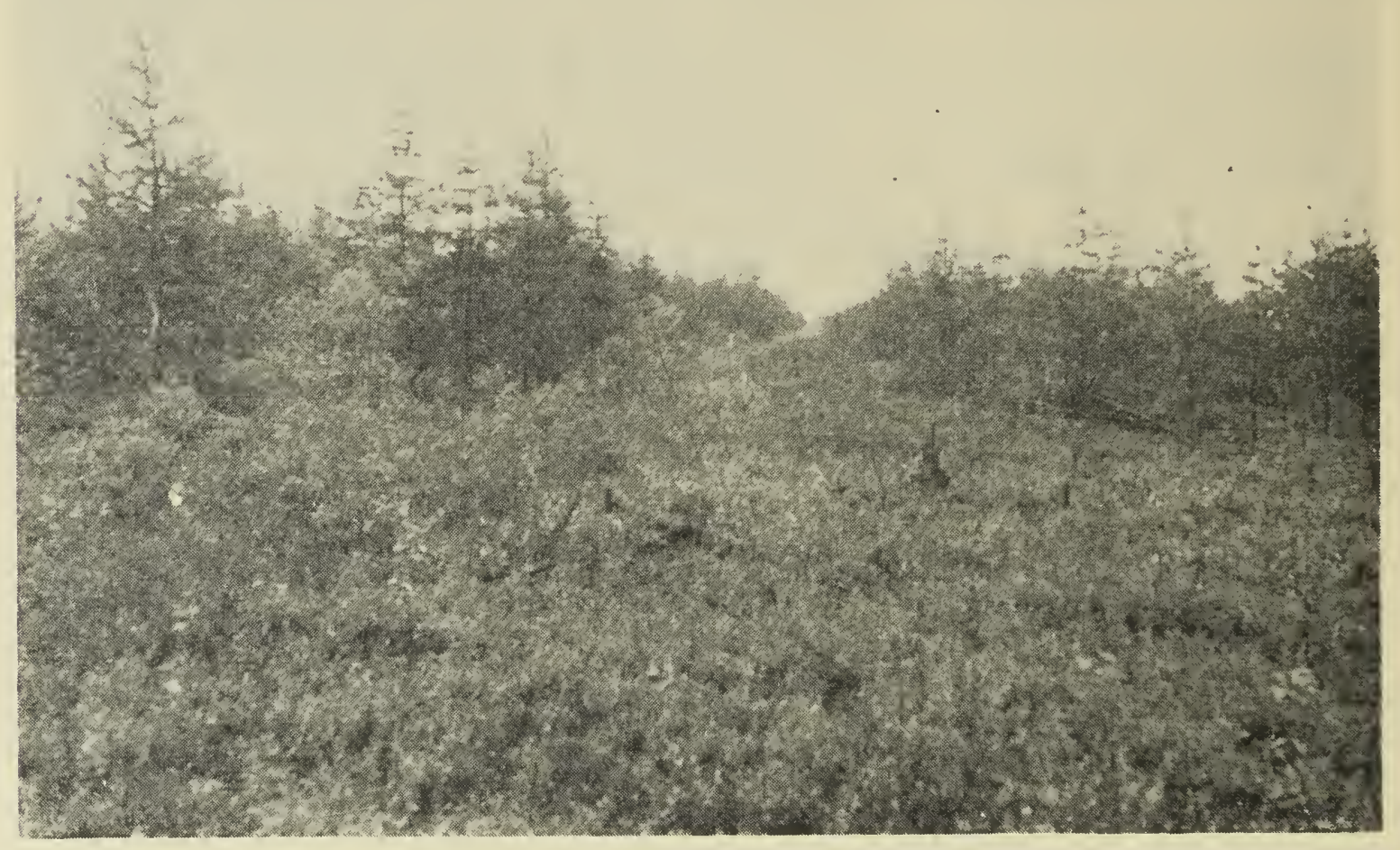

Fig. 13.-Coupe-feu artificiel séparant un terrain privé d'un terrain de la Couronne (à droite) brûlé au printemps. Photographié le 14 juillet. (Lac St-Jean).

Conclusion.-Il reste que le brûlage comporte de sérieux inconvénients. On a fait quelques tentatives pour en éliminer quelques-uns. Deux autres méthodes lui ont été comparées: $1^{\circ}$ fauchage au ras du sol $(3) ; 2^{\circ}$ fauchage, avec application de terre sur le chaume (4). Dans les deux cas, les observations et l'analyse statistique des résultats se sont prononcées en faveur du brûlage comme moyen de tailler le bluet. C'est cette denière méthode qui est admise, mais comme un mal nécessaire, en attendant qu'on en découvre de plus efficaces. 


\section{LE DÉSHERBAGE}

La taille par le feu ne suffit pas à maintenir la production de la bluetière. II laut encore éliminer les plantes qui naissent et croissent en même temps que le bluet et jouent contre lui le rôle des mauvaises herbes, d'où l'appellation généralement employée pour désigner les plantes indésirables de la bluetière.

Les mauvaises herbes.-Les mauvaises herbes les plus communes dans les champs de bluets du nord du Québec sont les suivantes et peuvent être groupées sous trois catégories:

I. Plantes qui croissent en colonies denses el finissent par prendre la place du bluet:

1. Crevard du moutons (parfois appelé bois-chaud), (dans les Provinces Maritimes, laurier), (Kalmia angustifolia L., Kalmia à feuilles étroiles). La plus répandue de toutes.

2. Thé du Labrador (Ledum groenlandicum Oeder).

3. Thé des bois (aux Iles-de-Madeleine, pommes de terre), (Gaultheria procumbens L.).

4. Rouget ou quatre-temps (Cornus canadensis L.).

II. Arbusles qui, par leur taille supérieure à celle du bluet, lui interceptent la lumière:

1. Aulne (vergne, verne), (Alnus incana (L.) Willd).

2. Bouleau (Betula L. spp.).

3. Saules (Salix L. spp.).

4. Peuplier, tremble (Populus L. spp.).

III. Autres plantes communes des "brûlés":

1. Bois de savane (Comptonia asplenifolia (L.) Gaertn), ou (Myrica asplenifolia Linn.), (Comptonie à feuilles d'Asplénie), très répandu.

2. Fausse herbe-à-la-puce (Apocynum androsaemifolium L.), (Apocyn à feuilles d'Androsème).

3. Bouquets rouges (Epilobium angustifolium L.), Epilobe à feuilles étroites.

Les plus nuisibles sont les aulnes, bouleaux, peupliers et saules, le bois de savane et le crevard de moutons.

Dommages causés.-Ces plantes font à la bluetière plus de tort qu'on semble le réaliser. Plusieurs font ombrage sur le bluet. Toutes tirent du sol à leur profit l'humidité et les éléments nutritifs essentiels. Toutes pourraient, abriter une multitude d'insectes nuisibles.

La concurrence des mauvaises herbes avec le bluet pour la lumière est sérieuse Le bluet est lui-même une plante héliophile (qui aime la lumière). Chandler et Mason (5) disent que "le bluet doit recevoir 80 pour' cent et plus de pleine lumière solaire chaque année pour produire un nombre normal de bourgeons à fruits et donner un bon rendement de fruits". Les mêmes auteur's rapportent, d'après des données receuillies au photomètre, que "les mauvaises herbes interceptent de 70 ì 80 pour cent de la lumière solaire".

Effet général du brûlage.-Sauf dans un cas particulier (voir, plus bas, contrôle du crevard du moutons), le brûlage n'est pas recommandé pour la destruction des mauvaises herbes. Pour plusieurs de ces dernières comme pour le bluet, le brûlage a l'effet d'une taille et stimule leur propagation. Caron (1) conduisit, en 1940, à L'Ascension, Lac St-Jean, une enquête dont, les conclusions appuient rel avancé. 
On a examiné l'effet de trois brûlages effectués sur des terrains différents en 1938 , 1939 et 19.10, sur le bluet, le crevard de moutons, le bois de savane et le thé dı Labrador. Il a été remarqué que "la végétation souterraine de bluets et des trois espèces concurrentes est très vigoureuse, que le feu ne l'affecte pas, mais qu'il agit comme un stimulant semblable à une taille énergique, comme le ferait un rasage complet. Dans nos expériences sur l'herbe à la puce, alor's que nous avons essayé de détruire des colonies avec une torehe à essence, dont le feu est beaucoup plus ardent que celui qui rase les bluets, nous avons trouvé qu'à un pouce de profondeur la végétation souterraine n’était pas affectée, alors que toute la partie aérienne était en cendres ou carbonisée. Nous pouvons facilement supposer que les espères qui nous occupent ici ne font pas autrement et que le feu agit sur elles comme il le fait pour les bluets, c'est-à-dire qu'il les stimule."

Méthodes de destruction. Les méthodes de destruction qui seront exposées dans (ce bulletin ont été expérimentées par la Station expérimentale agricole d'Orono. Maine (5). Aucune d'elles n'ayant été soumise à une vérification dans la provinere de Québec, i! est impossible de les y recommander d'une façon définitive.

1. Aulnes, bouleaux, saules, elc. Ces essences se reproduisent par leurs graines. Les tiges sortent de bourgeons situés au niveau de la couronne et poussent en talle. De toutes les mesures de contrôle essayées, la plus efficace est l'arrachage de la couronne entière. On peut obtenir un certain contrôle en coupant les tiges à la serpe, deux années de suite. Du gros sel ordinaire (chlorure de sodium) appliqué sur toute la surface coupée de la couronne donne d'excellents résultats.

On peut encore détruire les saules en les coupant d'abord, et en couvrant la souche de gros sel ou encore en l'arrosant de chlorate de calcium (1 livre environ par gallon d'eau). PRÉCAUTION: le chlorate de sodium ou de calcium étant un explosif, il constitue un danger d'incendie et ne doit être manipulé qu'avec beaıcoup de prudence.

2. Le bois de savane ou complonie. C'est le pire ennemi de la bluetière. Le bois de savane croîl en formations serrées, jusqu'à une soixantaine de plants par verge carrée. (Fig. 14). "L'odeur balsamique de cet arbuste, écrit Marie-Victorin (13), est l'un des charmes qui corrigent la tristesse des grands brûlés sablonneux du nord (du Québec). La propagation de l'espèce, en milieu libéré par le feu, est éminemment favorisée par l'extension de rhizomes particuliers, grêles, blancs ou rougeâtres. qui courent horizontalement, se fixent de distance en distance par la production de racines, et émettent des bourgeons qui deviennent des tiges aériennes."

Le meilleur moyen de se débarrasser de cette plante c'est de couper les tiges aériennes au milieu de l'été. Dans les champs de bluets du Maine où la comptonic est aussi très répandue, Hildreth a trouvé (5) qu'on pouvait affecter la reproduction de cette plante en la coupant entre le 2 mai et le 10 septembre, et avec le plus de succès à la fin de juillet et au début d'août. D'autre part, l'analyse biochimique des racines indiquerait que la réserve d'hydrates de carbone est à son minimum en juillet et qu'elle augmente rapidement en juillet et août. Ce qui expliquerait comment la coupe faite à la fin d'août est si peu efficace, les racines ayant eu le temps d'accumuler assez de nourriture pour alimenter de nouveaux bourgeons souterrains au printemps suivant.

3. Le crevard de moutons ou kalmia.-D'après les essais faits au Maine, Loujours, l'application d'un engrais chimique complet (1,000 livres à l'acre) et de sulfate de manganèse (500 livres à l'acre) après un brûlage d'automne aurait grandement réduit la vigueur du crevard de moutons et causé un jaunissement des feuilles de cette plante sans affecter les plants de bluets.

Le brûlage seul effectué en octobre pendant trois années consécutives réduit considérablement la population du crevard de moutons sans empêcher les bluets 


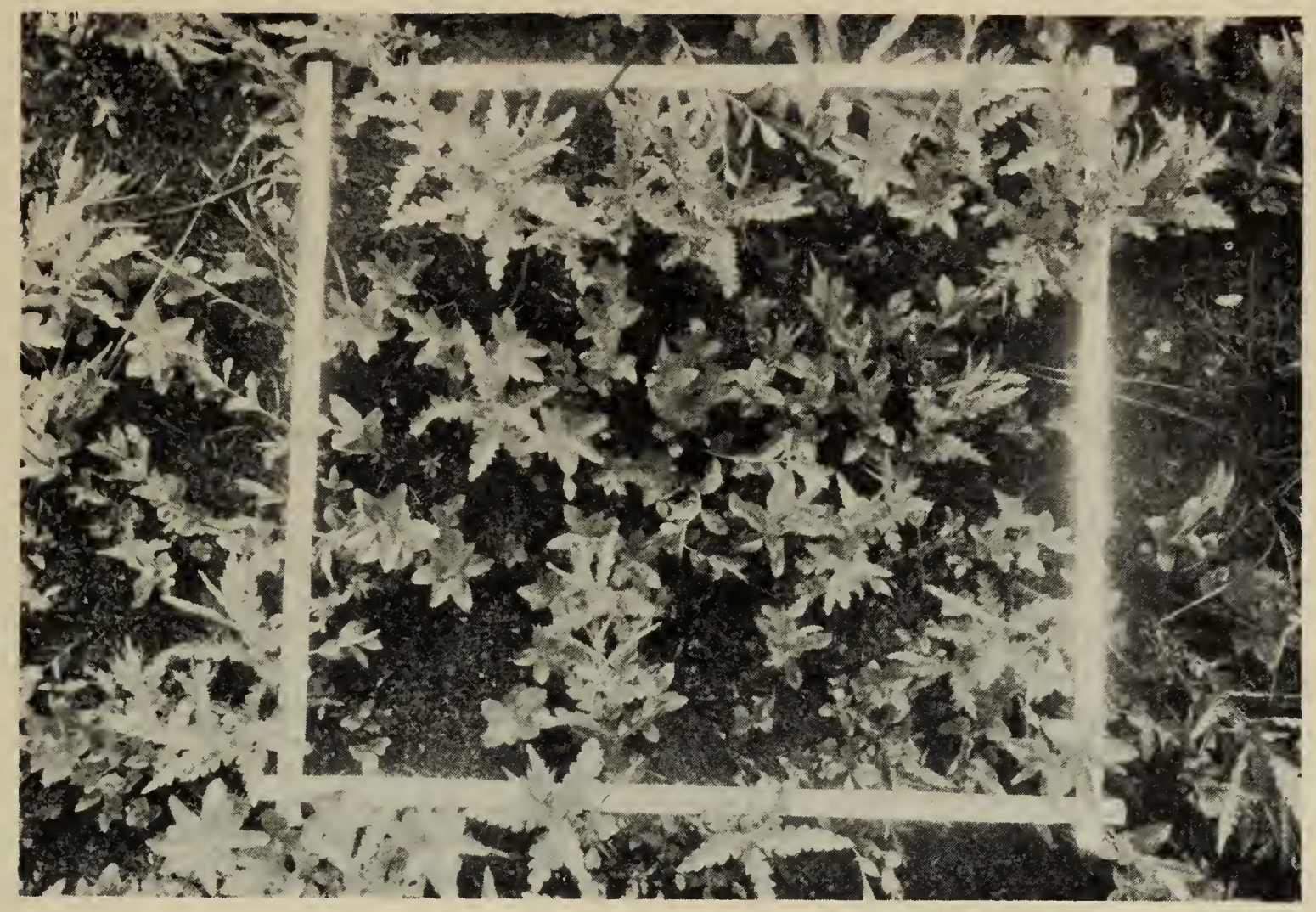

Fig. 14.- Le bois de savane ou comptonie, aux feuilles fortement dentelées, croît vigoureusement après un brûlage. Photographie prise le 14 juillet; brûlage effectué au au printemps de la même année. (Lac St-Jean).

de renaître ensuite plus denses, plus vigoureux et plus productifs. On atteindrait un résultat semblable en brûlant à tous les deux ans, à l'automne toujours, mais après une période beaucoup plus longue. On fait remarquer que cette pratique du brûlage fréquent est très dommageable au sol et ne devrait pas être prolongée. II faut aussi se rappeler que, sauf en cas d'éradication du crevard de moutons, l'engrais chimique ne doit pas être appliqué l'année même du brûlage.

4. Autres mauvaises herbes.-Les traitements recommandés contre les autres mauvaises herbes qu'on rencontre dans les bluetières du Québec sont les suivants:

a. Thé des bois: une solution de chlorate de sodium ou de calcium (1 livre par gallon deau) appliquée à plusieurs reprises sur le feuillage.

b. Rouget ou quatre-temps: application d'acide sulfurique (solution de 10 pour cent) ou de Dolge Weedkiller (1 pinte dans 20 pintes d'eau) ou chlorate de sodium (1 livre par gallon d'eau) en juin; traitement répété à la fin de juillet s'il se développe de nouvelles tiges.

c. Grande fougère: application d'acide sulfurique deux fois par année pendant deux ans; ou encore fauchage deux fois par année pendant deux ans.

d. Fausse herbe-à-la-puce ou apocyn: fauchage l'épété deux fois par année pendant deux ans; ou arrosage des feuilles avec du chlorate de calcium (1 livre par gallon d'eau).

e. Epilobe. Au Maine, cette plante fleurissant au moment où l'on effectue les traitements à l'arséniate de calcium contre les insectes, il y aurait danger d'empoisonner les abeilles qui la butinent. C'est pourquoi on a intérêt à faire disparaître cette plante. L'arrosage à l'acide sulfurique (solution de 10 pour cent) est efficace. 


\section{MALADIES ET INSEC'TES}

Bien qu'on puisse identifier certaines maladies du bluet dans les champs du Québec et des Provinces Maritimes, on y a rarement enregistré d'épidémies graves. Cel aspect de la production ne demande pas une attention immédiate dans la culture des bluetières.

Au Maine, la Station expérimentale d'Orono, ayant observé qu'un des facteurs limites de la production des bluets est la défoliation attribuable aux maladies du feuillage, a conduit une enquête rapportée en 1943 (14) établissant que le balai de sorcière, la rouille de la feuille. le mildiou poudreux (tache de la feuille) sont les maladies les plus communes. Moins répandues sont les suivantes: la feuille-rouge, la brûlure scléreuse de la tige, la brûlure de la fleur, la tache brune de la feuille. Toutes sont causées par des fungi et sont combattues au moyen de fongicides. (Voir, à ce sujet, la publication no. 754 intitulée "Le bluet", par le ministère fédéral de l'Agriculture).

Il est intéressant d'ajouter que, d'après Markin (14), le brûlage ordinaire ne semble pas éliminer complètement la feuille-rouge, bien qu'il la contrôle jusqu'à un certain point. Le brûlage ne contrôle pas le balai de sorcière. D'autre part, il semble refréner l'expansion de la tache de la feuille.

Le bluet du Québec ne souffre pas non plus, pratiquement, de dommages marqués de la part des insectes. Les fruits récoltés dans cette province n'ont jamais qu'on sache été refusés à la frontière pour une telle raison. C'est dans la NouvelleAngleterre surtout, et aux Provinces Maritimes, que la lutte contre les insectes est nécessaire. Ce sont le ver du bluet (Rhagoletis pomonella (Walsh), le charençon du gadelier (Pseudanthonomus validus Diets), le géomètre tacheté ou arpenteuse (Cingilia catenaria Drury) et autres. (On trouvera leurs descriptions et méthodes de contrôle dans la publication no. 754 du Ministère Fédéral de l'Agriculture).

Le brûlage (2) tient en échec la population du ver du bluet. L'insecte adulte (mouche) vient pondre ses oeufs sous la peau du fruit en voie de maturation. Au temps de la cueillette, la larve est déjà prête à quitter le fruit pour préparer ses quartiers d'hivernement à 2 ou 3 pouces sous la surface du sol. L'année suivante, elle émerge du sol à l'état adulte dès que les premiers bluets mûrs apparaissent, et se met à pondre. Un brûlage bien fait, en éliminant pour un an la récolte des bluets, empêche la propagation de l'insecte. On fait aussi remarquer que le contrôle de l'insecte est rendu plus difficile par la présence des mauvaises herbes qui lui offrent un abri.

L'emploi de l'arséniate de calcium contre le ver du bluet est courant en Nouvelle-Angleterre. On en fait deux applications successives à intervalles de 7 à 10 jours. La première fois, en juillet, après l'apparition de la mouche. Mais un intervalle de deux semaines doit séparer la deuxième application du début de la cueillette.

\section{FERTILISATION}

Le sol à bluet.--Le bluet nain pousse bien dans une grande variété de types de sols d'origines diverses, pourvu qu'ils soient très acides. Plus le sol est draîné, plus le plant est vigoureux. Le sol est généralement caractérisé par l'association d'essences forestières qui l'occupaient auparavant. Dans l'Etat de New Jersey (18), on a observé que le meilleur sol à bluet est celui qui porte du pin, du cèdre blane et de l'érable rouge, tandis qu'au Lac St-Jean, par exemple, le bluet succède à une forêt de pin gris (cyprès), d'épinette noire, de tremble, etc. 
Un profil typique du sol à bluet au Lac St-Jean a été étudié dans un terrain (incendié en 1941) où le bluet foisonne et où la ferme expérimentale de Normandin a commencé quelques recherches. En surface, une couche de 2 à 4 pouces d'épaisseur, constituée de débris végétaux, cendres et sable gris. Au-dessous, une couche de sable jaune ou jaunâtre sur une profondeur d'environ 2 pieds, qui repose ellemême sur une mince couche de sable gris, plus compacte, moins perméable. Enfin, encore plus profondément, un lit très épais de sables grossiers sur un fonds d'argile. L'acidité en surface varie autour du pH 4.5.

D'autres bluetières québécoises reposent sur un lit épais de débris organiques: savane du canton Racine (Lac St-Jean), plaine de la Rivière-Ouelle (comté de Kamouraska). On trouve aussi les bluets en abondance dans les sols rocailleux et les rochers acides, v.g. les "grands brûlés" de Kamouraska-sud.

$\mathrm{Au}$ Maine (2), le sol à bluet est généralement sableux-limoneux et pauvre en matière organique à cause des brûlages répétés. Ce sol repose sur une couche imperméable (hardpan) à quelques pouces ou quelques pieds sous la surface. Ces sols sont acides et leur $\mathrm{pH}$ varie de 4.4 à 5.7 .

La chaux.-Est-ce que la chaux endommage la bluetière? Chandler et Mason (6) en ont fait l'essai et ont conclu qu'une application d'une ou deux tonnes de chaux à l'acre peut augmenter sensiblement le rendement de la bluetière. Le bluet n'a pas semblé subir de dommages d'une application de 18 tonnes de chaux à l'acre répartie sur une période de cinq ans.

Engrais chimiques.-Le problème de la fertilisation des bluetières immenses où se fait la grande cueillette ne se pose même pas. Cependant, quelques propriétaires pourraient y songer pour augmenter le rendement en bluets sur ces sols trop légers ou trop rocheux dont ils ne sauraient espérer de rendements profitables en grains, racines ou légumes.

De toute évidence, il faut restituer au sol l'azote perdu au cours des brûlages et le phospore rendu inassimilable, ainsi que les éléments nutritifs enlevés par la récolte. Eaton (9) rapporte que "les producteurs de la Nouvelle-Angleterre prétendent avoir obtenu de bonnes augmentations de rendement au moyen d'engrais complets, mais les plantes concurrentes bénéficient également de ces engrais, et le rendement en bluets en souffre aussi longtemps que ces plantes ne sont pas complètement supprimées. Lorsque la terre est relativement débarrassée des autres plantes, on considère qu'une application, pendant la première ou la deuxième année de fructification, de 1,000 livres par acre d'engrais complet, comme le 5-9-8, est avantageuse. Il importe que l'engrais soit appliqué tôt au printemps, avant que les bourgeons s'ouvrent, car le feuillage de la plante est très sensible au contact des engrais chimiques".

\section{OUVRAGES CONSULTÉS}

(1) Caron, O., "Enquête botanique sur les lots de L'Ascension, Lac St-Jean, réservés à la culture des bleuets", Ministère de l'Agriculture, Québec (inédit).

(2) Chandler, F. B., "Lowbush Blueberries", Maine Agr. Exp. Sta. Bulletin no. $423,1943$.

(3) Chandler, F. B. et Mason, 1. C., "Pruning the Lowbush Blueberry", Proc. Amer. Soc. for Hort. Sci. 37: 609-610, 1940.

(4) Chandler, F. B. et Mason, I. C., "Pruning of Lowbush Blueberries", Proc. Amer. Soc. for Hort. Sci. 43: 173-174, 1943.

(5) Chandler, F. B. et Mason, I. C., "Blueberry Weeds in Maine and Their Control", Maine Agr. Exp. Sta. Bulletin no. 443, 1946. 
(6) Chandler, F. B. el autres, "Report of Progress", Maine Agr. Exp. Sta. Bulletins 363 (1932), 369 (1933) et 391 (1938).

(7) Darrow, G. M., Wilcox, R. B. el Beckwith, Chs. S., "Blueberry Growing", U.S.D.A., Farmer's Bulletin no. 1951, 1944.

(8) Eaton, E. L., "Culture of the Native Blueberry", Experimental Farms News, ler octobre 1945.

(9) Eaton, E. L., Eidt, C. C., Pickell, A. D. et Hockey, J. F., "Le Bleuet (arrellebleuet). I. La culture du bleuet (taton). II. La multiplication du bleuet (Fidt). IIt. Les insectes du bleuet et les moyens de les combaltre (Pickelt). IV. Les maladies du bleuet et leur traitement (Hockey). "Ottawa, pub. no. 751. (Bullelin du cullivateur no. 120), 1941.

(10) Johns, C. K., "Cooling Controls Invisible Mould in Blueberries", Canadian Food Industries, 18 (11): 22-23; 25, 1917.

(11) Kramer, A. et Shrader, A. L., "Significance of the pH of Blueberry Leaves", Plant Physiology, 20 (1): 30-36, 1945.

(12) Lathrop, F. H. et Nickols, C. B., "The Blueberry Maggot From an Ecological Viewpoint", Ann. Ent. Soc. Amer. 24 (2): 260-281, 1931.

(13) Marie-Victorin, Fs., "Flore Laurentienne”, Imprimerie de La Salle, Montréal, 1935.

(14) Markin, F. L., "Blueberry Diseases in Maine", Maine Agr. Exp. Sta. Bulletin no. $419,1943$.

(15) Munson, W. M., "The Blueberry in Maine" Maine Agr. Exp. Sta. Report 1898, pages 161-172, 1898.

(16) Munson, W. M., "The Horticultural Status of the Genus Vaccinium", Maine Agr. Exp. Sta. Bulletin no. 6, 1901.

(17) Rousseau, Jacques (Jardin Botanique de Montréal), Correspondance avec l'auleur', 1918.

(18) Slate, G. L. el Collison, R. C., "The Blueberry in New York", New York Slate Agr. Exp. Sla. Circulaire no. 189, 1940. 


\section{SERVICE DES FERMES EXPÉRIMENTALES}

Directeur, E. S. Archibald, B.A., B.S.A., LL.D., D.Sc.

Directeur associé, E. S. Hopkins, B.S.A., M.Sc., Ph.D.

Agriculteur du Dominion .................. O. Ripley, B.S.A., M.Sc., Ph.D.

Hortieulteur du Dominion. . . . . . . . . . . . . M. B. Davis, B.S.A., M.Sc.

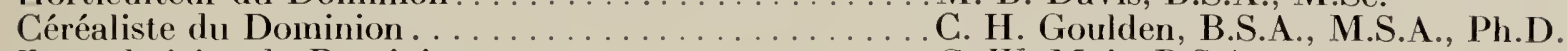

Zootechnicien du Dominion . . . . . . . . . . . . G. W. Muir, B.S.A.

Agrostologiste du Dominion . . . . . . . . . . . . . . . . M. Stevenson, B.S.A., M.Sc., Ph.D.

Aviculteur du Dominion. . . . . . . . . . . . . H. S. Gutteridge, B.S.A., M.Sc.

Chef, Division des tabacs. . . . . . . . . . . . . A. MacRae, B.A., M.Sc., Ph.D.

Apiculteur du Dominion . . . . . . . . . . . . C. B. Gooderham, B.S.A.

Surveillant en chef des stations de démonstration ...... J. C. Moynan, B.S.A.

Spécialiste en plantes textiles................ J. Hutchinson.

\section{ILE DU PRINCE-ÉDOUARD}

Régisseur, Station expérimentale, Charlottetown, R. C. Parent, B.S.A., M.Sc.

Régisseur, Renardière expérimentale, Summerside, C. K. Gunn, B.Sc., Pl.D.

\section{NOUVELLE-ECOSSE}

Régisseur, Ferme expérimentale, Nappan, W. W. Baird, B.S.A.

Régisseur, Station expérimentale, Kentville, A. Kelsall, B.S.A.

\section{NOUVEAU-BRUNSWICK}

Résisseur, Station expérimentale, Fredericton, S. A. Hilton, B.S.A., M.S.A.

\section{QUÉBEC}

Régisseur, Station expérimentale, Lennoxville, J. A. Ste-Marie, B.S.A.

Régisseur, Station expérimentale, Ste-Anne-de-la-Pocatière, J.R. Pelletier, B.S.A., M.A., M.Sc.

Régisseur, Station expérimentale, L'Assomption, R. Bordeleau, B.S.A.

Régisseur, Station expérimentale, Normandin, A. Belzile, B.S.A.

Préposé, Sons-station expérinentale, Ste-Clothilde, F. S. Browne, B.S.A.

Ferme expérimentale centrale, Ottawa.

\section{ONTARIO}

Préposé, Station expérimentale, Kapuskasing, M. B. Wianko, M.S.A.

Régisseur, Station expérimentale, Harrow, H. F. Murwin, B.S.A.

Préposé, Sous-station expérimentale, Delhi, F. A. Stinson, B.S.A., M.Sc.

Préposé, Sous-station expérimentale, Smithfield, D. S. Blair, B.S.A., M,Se.

\section{MANITOBA}

Régisseur, Ferme expérimentale, Brandon, R. M. Hopper, B.S.A., M.Sc.

Régisseur, Station expérimentale, Morden, W. R. Leslie, B.S.A.

Préposé, Pilot Flax Mill, Portage la Prairie, E. M. MacKey, B.S.A.

\section{SASKATCHEWAN}

Préposé, Ferme expérimentale, Indian Head, J. G. Davidson, B.A., B.S.A., M.S.A.

Régisseur, Station expérimentale, Scott, G. D. Matthews, B.S.A.

Régisseur, Station expérimentale, Swift Current, G. N. Denike, B.S.A.

Régisseur, Station expérimentale, Melfort, H. E. Wilson, B.S.A.

Régisseur, Sous-station expérimentale, Regina. J. R. Foster, B.S.A.

Régisseur, Pépinière forestière, Indian Head, John Walker, B.Sc., M.S.

Régisseur, Pépinière forestière, Sutherland, W. L. Kerr, B.S.A., M.Sc.

\section{ALBERTA}

Régisseur, Station expérimentale, Lacombe, G. E. DeLong, B.S.A., M.Sc. Régisseur, Station expérimentale, Lethbridge, A. E. Palmer, B.Sc., M.Se. Régisseur, Station expérimentale, Beaverlodge, E. C. Stacey, B.A., M.Sc. Préposé, Sous-station expérimentale, Fort Vermilion, V. J. Lowe.

Régisseur, Station herbagère expérimentale fédérale, Manyberries, H. F. Peters, B.S.A.

\section{COLOMBIE-BRITANNIOUE}

Régisseur, Ferme expérimentale, Agassiz, W. H. Hicks, B.S.A.

Régisseur, Station expérimentale, Summerland, R. C. Palmer, B.S.A., M.Se., D.Se.

Régissenr, Station expérimentale, Prince George, F. V. Hutton, B.S.A.

Régisseur, Station expérimentale, Saanichton, J. J. Woods, B.S.A., M.S.A.

liégisseur, Sons-station expérimentale, Smithers, W. 'T. Burns, B.S.A., M.Se.

\section{YUKON ET TERRITORRES DU NORD-OUEST'}

Préposé, Sous-station expérimentale, Whitehorse, T.Y., J. W. Abbott.

Préposé, Sous-station expérimentale, Fort Simpson, T.N.O., J. A. Gilb 
OTTAWA

EDMOND CLOUTIER, C.M.G.. B.A.. L.Ph.,

MMRIMEUR DE SA TRES EXCELLENTE MAJESTE LE ROI CONTROLLEUR DE LA PAPETERIE

19.18 